\title{
LA POLITICA COMPARATA: PREMESSE E PROBLEMI
}

\author{
di Giovanni Sartori
}

In un certo senso è vero che tutta la scienza politica sottintende, per quanto implicitamente, un quadro di riferimento comparato. Anche il politologo che esamina un caso singolo deve tener presente il cosiddetto contesto generale, o quantomeno dovrebbe tener presente altri casi. Altrimenti la sua analisi del caso singolo « esce di proporzione ». Senza dubbio è cosí. Ma questa è soltanto una verità banale.

Partiamo da questa premessa: che qualsiasi discorso si avvale di concetti che sono, a guardar meglio, «generalizzazioni camuffate ${ }^{1}$. A questa regola non fa eccezione nemmeno il discorso dello storico. Per quanto si sostenga che la conoscenza storica è conoscenza dell'individuale, $\mathrm{o}$ individuante, e che la storiografia non è una disciplina nomotetica, tutto ciò concesso resta vero che persino lo storico generalizza, lo voglia o non lo voglia. Ma se tutti generalizziamo in qualche modo e misura, il problema della validità delle nostre generalizzazioni si pone soltanto a coloro che generalizzano consapevolmente e di proposito. Il quesito è: come verificare, o falsificare, una generalizzazione? E il mio discorso comincia da qui. Sarà anche vero che non si può studiare la politica senza comparare. Ma la politica comparata si pone come tale solo quando le comparazioni, da implicite e casuali che erano, diventano esplicite e sistematiche.

Procedendo con ordine, la prima domanda è: perché comparare? La seconda domanda è: cosa è comparabile? Dopodiché si entra nel vivo del tema, e cioè possiamo passare alla domanda: come comparare?

1 Cosí Reinhard Bendix, Concepts and Generalizations in Comparative Sociological Studies, in «American Sociological Review », XXVIII (1963), p. 533. 
Controllo comparato e controllo storico.

Perché comparare? A cosa serve? La risposta è semplice: La comparazione è un metodo di controllo delle nostre generalizzazioni, previsioni o leggi del tipo «se... allora... ». Dico un metodo di controllo perché, ovviamente, non è il solo. Non è nemmeno un metodo di controllo «forte». Ma il metodo comparato ha dalla sua la forza di arrivare là dove altri strumenti di controllo non arrivano.

Grosso modo, le scienze dell'uomo si avvalgono di quattro strumenti o tecniche di verifica. Seguendo un ordine di « forza di controllo » decrescente, essi sono: 1) il metodo sperimentale, 2) il metodo statistico, 3) il metodo comparato, 4) il metodo storico ${ }^{2}$.

$\mathrm{Va}$ da sé che il metodo sperimentale è il piú sicuro, il piú soddisfacente. Ma è altrettanto intuitivo che questo metodo può essere utilizzato (con molta approssimazione) solo per problemi di micro-analisi. Largamente applicato in psicologia, è di difficile applicazione - in scienza politica e sociologia - al di là dell'ambito dei piccoli gruppi. Va anche da sé, in secondo luogo, che lo strumento statistico costituisce una tecnica di controllo da utilizzare ogni qualvolta sia possibile. A questo riguardo la difficoltà non è soltanto che occorrono grandi numeri. La ulteriore grossa difficoltà è che in materia sociale e politica $i$ dati suscettivi di trattamento statistico sono di sospetta attendibilità e validità ${ }^{3}$.

La comparazione è dunque il metodo di controllo sul quale siamo costretti, il piú delle volte, a ripiegare. Quando l'esperimento è impossibile, e quando mancano dati pertinenti e sufficienti per un trattamento statistico, non abbiamo scelta: idobbiamo verificare (o falsificare) comparando. $\mathrm{Da}$ quanto sopra si evince altresí che non dobbiamo confondere il «metodo comparato » con la « comparazione statistica ». E chiaro che anche con i dati statistici facciamo, e possiamo fare, delle comparazioni. Ma una cosa sono le regole di controllo statistico, e altra cosa sono le regole di controllo comparato. Possiamo anche metterla cosí: quando parliamo di metodo com-

${ }^{2}$ Cfr. Arend Lijphart, Il metodo della comparazione, in questo fascicolo. Peraltro l'A. sviluppa soltanto le prime tre voci.

${ }^{3}$ Per il primo punto rinvio alle osservazioni di Lijphart, loc. cit. Il secondo punto - la dubbia validità - verrà approfondito infra ai par. 4.5. 
parato è inteso che affrontiamo problemi non risolvibili in chiave statistica.

Il metodo storico viene ultimo nella mia lista perché è il piú debole. Tanto debole, ai fini del controllo, che molti sociologi e politologi non lo mettono nemmeno in conto. $\mathrm{Ma}$ in tal caso sbagliano, o sono irriconoscenti. Dopotutto la storia è un immenso deposito di esperienze, esperienze (non esperimenti) dalle quali ricaviamo, o possiamo ricavare, conferme o smentite. In linea di principio, dunque, «negare la storia » è assurdo, è autolesionismo. Il problema non è se la storia sia una preziosa fonte di dati alla quale attingere: lo è. Le perplessità sorgono sul come, e cioè su come utilizzare il materiale storico ai nostri fini, che sono - ricordiamolo - fini di controllo.

Sia chiaro: una cosa è il metodo storiografico di cui si avvale lo storico per conoscere la storia, e cioè per fare storiografia. Tutt'altra cosa è il controllo storico che interessa al politologo per fare scienza politica (e magari al sociologo per fare sociologia). Il politologo non è uno storico, e non fa storiografia. A lui interessa soltanto il controllo storico, e cioè un «trattamento della storia » atto a verificare delle leggi ovvero a generare ipotesi generalizzanti. Ma perciò ogni polemica tra storici e politologi è malposta. Al massimo lo storico potrà dubitare che il politologo riesca nella sua impresa. Sul che possiamo anche convenire. Difatti - dicevo il controllo storico è il metodo di controllo piú debole, quello che offre meno garanzie. E importa capire perché.

Si prenda Gaetano Mosca, che rappresenta assai bene la fase pre-scientifica della scienza politica. Se a Mosca si rimprovera di usare, come elemento prova, la storia, gli si rivolge un rimprovero infondato. La storia può « insegnare », come diceva Mosca ${ }^{4}$. E può anche provare. Il guaio è che il controllo storico di Mosca è senza metodo: consiste in una sequela di esempi a caso, presi alla rinfusa. Con questo nonmetodo si può provare tutto: perché nulla vieta a un autore di tacere tutti $i$ casi storici che non quadrano con la legge che gli interessa di provare. Il che non è detto per Mosca,

${ }^{4}$ Cfr. Ciò che la storia potrebbe insegnare, che è il saggio introduttivo che dà anche il titolo alla raccolta di scritti minori di Mosca edita da Giuffrè, Milano, 1958. Ma vedi Elementi di scienza politica, Bari, Laterza, 1939, vol. I, cap. I, spec. p. 69. 
il quale usò con intelligenza e competenza gli elementi di prova che allora esistevano e nei quali allora si credeva. $\mathrm{Ma}$ cosí come sarebbe antistorico giudicare Mosca con i criteri del nostro tempo, è egualmente antistorico giudicare il nostro tempo con i criteri di Mosca. E dunque importa affermare che, oggi, il metodo storico di Mosca (e di Pareto) non può essere accettato come un «controllo storico » atto a soddisfare i canoni di un controllo scientifico.

Quali sono, allora, le difficoltà? Conviene vederle alla luce della differenza tra metodo storico e metodo comparato. Il controllo comparato si fa, di solito, lungo uno spaccato orizzontale, e cioè in termini sincronici. In politica comparata confrontiamo quasi sempre unità geo-politiche, oppure processi e istituzioni, in un tempo eguale, o meglio dato per eguale. Cosí facendo, scontiamo la variabile «tempo ». Questa semplificazione non è senza peccato, soprattutto quando studiosi sprovveduti non avvertono che una cosa è il sincronismo «cronologico » (del calendario), e tutt'altra cosa il sincronismo «storico » (di tempi storici equivalenti). Resta il fatto che la comparazione sincronica semplifica di molto i problemi, perché autorizza un largo uso della clausola ceteris paribus. Se i tempi sono davvero eguali (cioè se sono tempi storici abbastanza equivalenti), allora è lecito presumere che tutta una serie di condizioni sottintese siano simili. Con il che le possiamo scontare.

Il controllo storico, per contro, è tale proprio perché mette in questione uno spaccato verticale, e cioè diacronico. Se il metodo comparato si dispiega orizzontalmente, il metodo storico investe invece, caratteristicamente, una dimensione longitudinale. Dal che sembra conseguire che non possiamo piú postulare la «parità delle condizioni »: semmai, il piú delle volte dobbiamo presumere un ceteris non-paribus. Il che introduce una complicazione, una grossa complicazione, al posto di una vantaggiosa semplificazione.

Questa prima differenza - si obietterà - non dimostra ancora che il controllo storico sia piú debole di quello comparato: dimostra soltanto che il primo è piú arduo del secondo. Verissimo. $\mathrm{Ma}$ è altrettanto vero che nella misura in cui quelle maggiori difficoltà non sono superate, nella stessa misura il controllo storico resta un controllo piú imperfetto, o meno soddisfacente, di tutti gli altri. Conclusione rafforzata da una seconda differenza. 
La seconda differenza investe la reperibilità dei dati. In politica comparata lamentiamo, giustamente, che $\mathrm{i}$ dati che ci occorrono non ci sono, o che sono insufficienti e inadeguati. Il che è indubitabile. Ma siccome lavoriamo « al presente», nulla vieta - in linea di principio - che i dati mancanti vengano rilevati. Difettiamo sí di informazioni: ma siamo ancora in tempo a cercarle. La documentazione storica, invece, è quella che è. Non è soltanto questione di tornare a frugare negli archivi con nuovi occhiali. La questione è che ogni epoca registra se stessa per come si vede, con le sensibilità e gli interessi conoscitivi che ha. A questo effetto, dunque, il controllo storico si imbatte in un ostacolo che risulta insuperabile, e che certo ne limita grandemente l'applicabilità. Questa volta si tratta davvero di un limite in re.

Accenno di passata a un ulteriore problema, o meglio a un elemento di perplessità che giro — per la risposta - agli storici. Assistiamo ad un vertiginoso crescendo di accelerazione storica, accelerazione che mette in crisi il postulato di continuità sul quale ha sinora poggiato - piú o meno consapevolmente - la storiografia di tutti i tempi. Allo storico si pone sempre piú un problema inedito di filosofia della storia. Historia facit saltus. Quantomeno, tra le generazioni la saldatura si allenta e il distacco aumenta: tanto da lasciar sospettare che la storia proceda oramai «per stacchi » (il che non è lo stesso che dire dialetticamente, o avversativamente). Quanto pesa, allora, il passato sul presente? Qual è l'elemento di continuità nella discontinuità? Esiste un « uomo perenne » capace di sfuggire all'usura di qualsiasi velocità di cambiamento? Non pretendo di rispondere. Sollevo questi interrogativi soltanto per notare che il metodo storico avrebbe piú titolo per raccomandarsi alle scienze sociali se gli storici rimettessero in regola le proprie credenziali. Finora gli storici hanno avuto ragione nell'accusare $i$ sociologi, e in parte $i$ politologi, di ignoranza storica. Ma se faranno finta di non vedere che l'accelerazione storica costituisce una «variabile perturbatrice » con la quale debbono fare $\mathrm{i}$ conti, saranno gli storici a rischiare una crisi di credibilità.

$\mathrm{Ma}$ vale concludere con alcuni spunti costruttivi. Il primo spunto mi viene da Lijphart quando avverte che anche il case study, lo studio di un caso singolo, può essere concepito e utilizzato - dal comparatista - come uno strumento di controllo di ipotesi. Anche lo studio del caso, egli dice, può 
generare ipotesi e servire a verificarle ${ }^{5}$. Vorrei aggiungere che questo argomento è perfettamente applicabile ai casi storici (e non soltanto a casi ancora da studiare). Posto uno schema teorico, e per esso delle leggi o generalizzazioni, possiamo benissimo reperire una documentazione storica sufficiente per vagliarle alla luce di «casi passati » opportunamente isolati e ricostruiti.

Un altro settore di utilizzazione del metodo storico riguarda la teoria che può essere detta, per intendersi, delle sequenze e dei sovraccarichi. Lo sviluppo politico è stato analizzato da molti politologi come una sequela di « crisi », di grossi nodi da sciogliere. Se queste crisi - si sostiene - sono abbastanza distanziate l'una dall'altra, e se seguono un determinato « ordine di sequenza », in tal caso il sistema politico le può digerire, le può risolvere. Invece se queste crisi si cumulano, e esplodono in un ordine di sequenza sbagliato, allora abbiamo un «sovraccarico » ed è il sistema politico che entra in crisi. $\mathrm{E}$ evidente che questo è un discorso longitudinale. E tanto evidente che la teoria in questione viene desunta dall'esperienza storica e che richiede il sostegno di prove storiche. Lo schema delle crisi - crisi di secolarizzazione, di legittimità, di suffragio, di industrializzazione, di penetrazione, di urbanizzazione, di distribuzione, e simili — è piú o meno delineato ${ }^{6}$. La difficoltà - ai fini del controllo storico - è una difficoltà di " periodizzazione ». Se la definizione di ciascuna di queste crisi non è convertibile in una adeguata delimitazione storica, e cioè in un soddisfacente periodizzamento, la tesi resta campata in aria. Per esempio: come si periodizza - per stabilire equivalenze tra paese e paese - la cosiddetta crisi di industrializzazione? Ecco uno dei tanti interrogativi che si pongono alla teoria dello sviluppo politico, e che richiedono, per essere affrontati e risolti, il controllo storico.

Non occorre dilungarsi in altri esempi di come il politologo e il sociologo trattano la storia ai loro fini. Ricordo sol-

5 Lijphart, Il metodo della comparazione, loc. cit.

6 Per una applicazione della dottrina delle crisi e dei sovraccarichi ai nostri sistemi democratici, specie per le crisi di legittimità e di suffragio, cfr. Domenico Fisichella, Sviluppo democratico e sistemi elettorali, Firenze, Sansoni, 1970, pp. 14-44. Con riferimento invece al terzo mondo vedi Lucian Pye, Aspects of Political Development, Boston, Little, Brown, 1966, pp. 62-67; e ancor piú il VII vol. della serie «Political Development 》 edito dalla Princeton University Press: AA.VV., Crises in Political Development, di prossima pubblicazione. 
tanto i nomi di Eisenstadt, di Lipset e Rokkan, e l'ultimissimo Almond ${ }^{7}$. Non c'è dubbio: non dobbiamo né possiamo rinunziare al controllo storico. Anche se - per le ragioni che si sono viste - si tratta di un controllo difficile e insicuro, integrativo e non certo sostitutivo dei metodi di controllo piú forti ${ }^{8}$.

\section{Cosa è « comparabile»?}

$\mathrm{Al}$ quesito «perché comparare? » ho risposto che la comparazione è il meno insoddisfacente tra i metodi di controllo accessibili; accessibili, beninteso, alla scienza politica, e soprattutto al politologo impegnato in problemi di macro-analisi. Resta un secondo quesito preliminare: cosa è comparabile? Diciamo spesso che una certa comparazione è sbagliata, e che è sbagliata perché non si può fare. Non ha senso paragonare sassi e pere, e forse nemmeno uomini e cavalli. A lume di naso, sta bene. Ma quali sono i criteri in ordine ai quali dichiariamo due o piú cose paragonabili, ovvero non-paragonabili? Quand'è che due o piú fattispecie possono essere confrontate, oppure no?

Molti autori dichiarano che comparare equivale a «assimilare », nel senso che la comparazione si fonda su operazioni di assimilazione, sul rendere simile il dissimile. Ma questa tesi legittima qualsiasi arbitrio. A questa stregua, con un po' di virtuosità verbale si può accoppiare tutto, o quasi tutto. Difatti la tesi viene sovente corretta, o frenata, osservando che non si tratta di «inventare » somiglianze (fittizie) ma di «scoprire» somiglianze (in re). Ma la diga è fragile,

7 Tra i molti scritti di Shmuel N. Eisenstadt ricordo due concisi saggi: Initial Institutional Patterns of Political Modernization, in «Civilizations », IV (1962), I (1963); Institutionalization and Change, in "American Sociological Review », XXIX (1964). Seymour Lipset e Stein Rokkan sono richiamati per la loro Introduzione al vol. coll. Party Systems and Voter Alignments, New York, Free Press, 1967, tutta in chiave di sequenze longitudinali. L'ultimissimo Almond è quello che cerca di « integrare » sincre tisticamente i vari approcci, ivi includendo quello storico. Vedine Determinacy-Choice, Stability-Change - Some Thoughts on a Contemporary Polemics in Political Theory, in "Government and Opposition 》, V (196970), pp. 22-40.

8 In generale, cfr. Sylvia L. Thrupp, Diacronic Methods in Comparative Politics, in R. T. Holt e J. E. Turner (eds.), The Methodology of Comparative Research, New York, Free Press, 1970. 
la differenza sottile. $\mathrm{E}$ per mio conto non accetto la premessa che paragonare equivale ad assimilare. Questa impostazione è quantomeno fuorviante, e deve essere rettificata.

In primo luogo, non. sta scritto in nessun testo sacro che il comparatista debba cercare somiglianze invece che differenze. Inoltre, e in ogni caso, le due operazioni sono complementari. Per trovare una somiglianza bisogna isolarla da tutto ciò che simile non è. Vale a dire, il simile deve essere « estratto » dal dissimile. Se le differenze non sono ben individuate, le somiglianze rischiano di essere fraudolente e pasticciate.

Una tesi piú tecnica, o sofisticata, è questa: che noi dichiariamo simili le caratteristiche che vogliamo mantenere costanti (e cioè trattare come parametri), talché il dissomigliante è dato dalle caratteristiche che vogliamo trattare come variabili, e cioè che lasciamo variare. $\mathrm{Ma}$ questa è una sottotesi, per dire che essa vale soltanto per taluni aspetti di una fase avanzata di manipolazione dei dati. Chi si contenta di una risposta del genere è chi non vede il problema a monte, o chi cerca di eluderlo.

Ripetiamo la domanda: quali sono i criteri che consentono di dichiarare due o piú cose (o attributi) paragonabili, e cioè tali da poter essere trattate insieme? Insisto nel dire « criteri » perché la politica comparata può crescere additivamente, o cumulativamente, solo a patto di stabilizzare - in qualche modo - il trattamento delle similarità e delle dissimilarità. I criteri sono, appunto, regole impersonali, e come tali un elemento oggettivo, stabilizzante. E se cerchiamo dei criteri, questi criteri sono, a tutt'oggi, soltanto quelli forniti dalla logica classificatoria, dalla analisi per genus et differentiam.

Pensiamoci bene. Quando asseriamo che certe cose o caratteristiche sono comparabili, quel che sottintendiamo è che appartengono allo stesso genere, specie, o sotto-specie: insomma, che appartengono ad una stessa « classe ». Viceversa, quando sosteniamo che due o piú cose non sono paragonabili, quel che sottintendiamo è, appunto, che sono « eterogenee », e cioè che non appartengono allo stesso genere. La comparabilità rinvia, allora, alla omogeneità. Per contro, la non-comparabilità è posta dalla eterogeneità. Beninteso, omogeneità e eterogeneità non sono « in natura »; sono classi fabbricate dalla logica classificatoria, e per essa dai nostri criteri di classificazione. Nondimeno il fondamento tassonomico della paragonabilità pone un formidabile 
freno all'arbitrio soggettivo. Chi si avventura in paragoni azzardati si imbatte nella perentoria obiezione dell'alienum genere. $\mathrm{E}$ per superare questa obiezione l'onere della prova non è lieve. A chi mette a confronto l'incomparabile (tale alla luce delle classificazioni in uso) incombe il dovere di costruire una nuova classificazione dalla quale risulti la omogeneità di quel che altrimenti risultava eterogeneo.

Facciamo il punto. «Comparabile » sta per dire: cose che appartengono allo stesso genere, specie, sottospecie, e cosí via. Pertanto l'elemento di similarità che legittima le comparazioni è l'identità di classe. Correlativamente, le dissimilarità sono poste - in via primaria - da quel che differenzia le specie dal loro genere, le sottospecie dalla loro specie, e, in generale, ogni sotto-classe dalla sua classe di appartenenza. E vero che registriamo anche altre differenze. $\mathrm{Ma}$, come vedremo, queste ulteriori differenze sono secondarie, nel senso che si limitano a registrare le variazioni interne di una stessa classe.

Fin qui stiamo sul sicuro. Intendo dire che nella misura in cui facciamo capo alla logica classificatoria, al trattamento « per genere prossimo e differenza specifica », nella stessa misura sappiamo stabilire che cosa è comparabile, e cosa non lo è. Senonché - si obietterà - le tassonomie e la logica classificatoria stanno passando di moda. Ma se cosí è, occorre sostituire quel che ripudia. Altrimenti - e cioè in difetto di una alternativa adeguata - le nostre comparazioni rischiano di oscillare perigliosamente tra due estremi: un eccesso di assimilazione (che vanifica il controllo), e un eccesso di differenziazione (che vanifica la comparazione). Come vedremo in seguito.

La comparazione globale.

L'interesse per la politica comparata nasce quando allunghiamo lo sguardo extra moenia. Dico l'interesse, perché « allargare le conoscenze » non è ancora comparare. Non è detto che chi studia « piú di un solo paese » sia per ciò stesso un comparatista. Tale era indubbiamente Carl Friedrich quando pubblicava, nel 1937, la prima versione del suo classico studio su Constitutional Government and Democracy ${ }^{9}$. Tale era anche, negli stessi anni, Herman Finer, il cui trattato Theory and Practice of Mo-

9 Il titolo originario del 1937 era Constitutional Government and Politics. Il libro, quasi interamente riscritto nel 1941, è stato ripubblicato 
dern Government resta un classico della scuola istituzionale ${ }^{10}$. $\mathrm{Ma}$ tali non erano, e non sono, quegli autori che si limitano a studiare in giustapposizione, l'uno accanto all'altro, due o piú paesi. Nulla vieta, ovviamente, che un gruppo di paesi venga analizzato partitamente. $\mathrm{Ma}$ in tal caso occorre almeno uno schema concettuale unitario, una analisi condotta, per ogni paese, con lo stesso metro ${ }^{11}$.

Non occorre riepilogare i vari sviluppi, e il rapido crescendo di consapevolezza comparata che ha caratterizzato soprattutto l'ultimo decennio ${ }^{12}$. A me interessa soltanto, in questa sede, il piú audace e il piú innovativo di questi sviluppi: il tentativo di costruire - mediante lo strumento comparato - « una teoria probabilistica della politica ${ }^{13}$. Questo tentativo, che fa soprattutto capo ad Almond e alla scuola dello sviluppo politico ${ }^{14}$, si

di recente in una $4^{\mathrm{a}}$ ed. aggiornata (Waltham, Blaisdell, 1968). La trad. it. Governo costituzionale e democrazia (Vicenza, Neri Pozza, 1950), davvero pessima, è condotta sulla ed. del 1950.

${ }^{10} \mathrm{Cfr}$. spec. l'ed. condensata in un volume del 1950, ripubblicata nel 1954 da Methuen, London. L'opera è stata ripresa in The Major Governments of Modern Europe, London, Methuen, 1961.

11 Un esempio particolarmente riuscito di questa impostazione è dato dai due volumi curati da Roy C. Macridis e Robert E. Ward, Modern Political Systems: Europe, e Modern Political Systems: Asia, Englewood Cliffs, Prentice-Hall, 1963. Pur essendo i singoli paesi affidati a nove autori, l'opera è unitaria. Lo è assai meno, per contro, il vol. coll. curato da $\mathrm{S}$. H. Beer e A. B. Ulam, Patterns of Government: The Major Political Systems of Europe, New York, Random House, 2a ed. 1962. Nonostante i buoni propositi, i collaboratori vanno in ordine sparso.

12 Per il rapido crescendo di consapevolezza comparata (in generale) basti questa indicazione quantitativa: la bibliografia commentata di Robert M. Marsh, Comparative Sociology, New York, Harcourt, Brace, 1967, raccoglie - per il periodo 1950-1963 - oltre 1100 titoli. Per il riepilogo degli sviluppi che sono propriamente di politica comparata cfr. l'Introduzione di Harry Eckstein all'antologia (da lui curata con David Apter) Comparative Politics. A Reader, New York, Free Press, 1963. E vedi, da ultimo, Jean Blondel, An Introduction to Comparative Government, New York, Praeger, 1969; e Samuel E. Finer, Comparative Governement, London, Allen Lane Penguin Press, 1970.

${ }_{13}$ Cosí Gabriel Almond, nella introduzione a The Politics of the Developing Areas, Princeton, Princeton University Press, 1960, p. 4.

14 Per la scuola dello sviluppo politico si deve segnalare la serie dei volumi sul « Political Development » promossa dal Social Science Research Council, e pubblicata - tra il 1963 e il 1966 - dalla Princeton University Press. In merito, e la bibliografia, vedi Fred W. Riggs, The Theory of Political Development, nel vol. coll. curato da James C. Charlesworth, Contemporary Political Analysis, Free Press, New York, 1967, spec. pp. 317-337; trad. it. Il Mulino, di prossima pubblicazione; e, piú 
presenta come una renovatio ab imis. In quanto tale merita di essere identificato con l'etichetta di «nuova » politica comparata. Sia chiaro: il nuovo non è necessariamente migliore di quanto gli preesiste, e dunque l'etichetta non ha - almeno per me - nessun sottinteso apprezzativo. Distinguere tra nuovo e meno nuovo (o tradizionale) è, sovente, solo un modo sbrigativo di orientarsi. Ma nel caso in questione si tentano vie nuove anche perché avvengono, e vengono registrate, grosse novità. $\mathrm{Ne}$ ricordo due: 1) l'ingrandimento, o espansione, della politica, e 2) la globalità, o globalizzazione della politica.

1) L'espansione della politica. La politica diventa «piú grande » per due rispetti, oggettivo e soggettivo. Oggettivamente parlando assistiamo ad un crescendo di politicizzazione, correlativo alla crescente penetrazione e diffusione dei credi politici, delle ideologie. Da un lato aumenta la sfera dell'intervento dello Stato; dall'altro aumenta la mobilitazione, o la partecipazione, delle masse. Viviamo dunque in un mondo sempre piú intriso e pervaso di politicità. Inoltre, dicevo, la politica si ingrandisce anche in chiave soggettiva, e cioè perché la vediamo in maniera allargata. La politica non coincide piú con la dottrina dello Stato. Oggi includiamo nella nozione di politica una « periferia » che prima veniva dichiarata extra-politica. Siamo anzi arrivati all'eccesso di studiare tutto quel che manifesta una 《 potenzialità politica ${ }^{15}$. Il che è davvero tutto, ed è troppo ${ }^{16}$.

2) La politica diventa, alla lettera, globale. Non è solo che l'eurocentrismo ha fatto il suo tempo; è che l'area occidentale non è piú l'epicentro di un mondo che è diventato policentrico. Ovviamente la politica comparata è la disciplina piú impegnata in questa apertura globale. Dagli studi intra-area, interni ad

ampiamente, Gianfranco Pasquino, Modernizzazione e sviluppo politico, Bologna, Il Mulino, 1970.

${ }^{15}$ Cosí Macridis, Comparative Politics and the Study of Goverment, in « Comparative Politics», I (1968), p. 81.

${ }^{16}$ Le riserve di Macridis sulla « graduale sparizione della politica » e su quella che chiama fallacy of inputism (spec. pp. 84-87, art. cit., supra) non sono dissimili dalle mie riserve sulla sociologizzazione della politica sviluppate in Alla ricerca della sociologia politica, in «Rassegna Italiana di Sociologia », IV (1968); riserve riprese, in parte, nella mia introduzione Per una definizione della scienza politica alla Antologia di scienza politica, Bologna, Il Mulino, 1970, spec. pp. 12-17. Vedi anche Glenn D. Paige, The Rediscovery of Politics, nel vol. coll. curato da J. D. Montgomery, W. J. Siffin, Approaches to Development. Politics, Administration and Change, New York, McGraw Hill, 1966, pp. 49-58. 
un'area (il piú delle volte l'area occidentale) si passa, o tenta di passare, a studi cross-area, inter-area. E un passo lungo, costellato di difficoltà. $\mathrm{Ma}$ occorre tentarlo anche per una ragione metodica. Quanto piú avvertiamo che la comparazione è uno strumento di controllo, e quanto piú consapevolmente « compariamo per controllare ", tanto meno possiamo escludere dei casi. Non è solo che piú casi ci sono, meglio è; è anche, e soprattutto, che un controllo lacunoso non è un buon controllo.

Il salto nella globalità è davvero un grosso salto. Supponendo che le unità considerate siano gli Stati, nel 1946 ne esistevano circa 80 . Non è improbabile che arriveremo presto a 150 Stati indipendenti, o almeno giuridicamente riconosciuti come tali. E questo numero dice il meno ${ }^{17}$. Per dire il piú occorre guardare alla straordinaria varietà $\mathrm{e}$, spesso, fluidità di questa proliferazione di entità politiche. Il corrispettivo della globalità è, in effetti, uno straordinario allargamento dello «spettro dei sistemi politici $»^{18}$. In questo spettro troviamo anche - nella orizzontalità sincronica - una longitudinalità diacronica, vale a dire sistemi politici che appartengono a stadi diversi di consolidamento e di strutturazione: sistemi embrionali e indifferenziati, non meno che sistemi straordinariamente sedimentati e complicati (quantomeno per l'osservatore occidentale).

Era inevitabile, anzi era necessario, che queste novità retroagissero sulla scienza politica in generale, e sulla politica comparata in particolare. Il problema di rivedere il proprio apparato concettuale è, oggi, un problema generale di tutta la scienza politica. Ma l'esigenza di « riconcettualizzare » - non solo di riesaminare, ma anche di inventare ex novo le proprie categorie - si pone in specialissimo modo in sede di politica comparata. E soprattutto la politica comparata che ha perduto le vecchie frontiere, e che deve cercare la «nuova frontiera ». Basti questa considerazione: che una politica comparata globale non è possibile senza categorie, o concetti, capaci di viaggiare, di viaggiare al di là dell'area occidentale. Laddove è chiaro che il nostro vocabolario politico è, in grandissima misura, un vocabolario che riflette le esperienze del mondo occidentale e fatto per inter-

17 Anche se il solo problema di standardizzare il conteggio non è piccolo. Cfr. B. M. Russett, J. D. Singer, M. Small, National Political Units in the Twentieth Century: A Standardized List, in «American Political Science Review », LII (1968), pp. 932-951.

${ }^{18}$ Cosí Ralph Braibanti, Comparative Political Analytics Reconsidered, in « Journal of Politics », XXX (1968), pp. 36-37. 
pretare quel mondo che ha fabbricato.

Questo sforzo di riconcettualizzazione viene sovente frainteso. La nuova scienza politica viene accusata di usare neologismi per ridire cose già dette, e di avvolgersi nella innecessaria oscurità di un gergo esoterico. Indubbiamente gli abusi ci sono. Il mondo pullula di intellettuali i quali, non essendo originali nel pensiero, fingono di esserlo in altro modo. E l'ondata del « novitismo » - del voler essere nuovi ad ogni costo — non risparmia nessuno, e nemmeno la scienza politica.

Ma gli abusi non tolgono che l'esigenza della riconcettualizzazione sia valida. Non si può negare, in primo luogo, che il vocabolario della politica sia altamente insufficiente, e che il progresso di ogni conoscenza scientifica vada di pari passo con la costruzione di un vocabolario piú preciso e articolato. In secondo luogo non è detto che si debbano inventare nuovi nomi soltanto per le cose innominate. Chi sostiene questa tesi non è aggiornato in semantica. Ogni parola suggerisce anche un modo di percepire, un modo di interpretare. Pertanto due o piú parole che hanno la stessa denotazione (che stanno per la stessa cosa) non hanno per questo la stessa connotazione. Non è lecito dichiarare un neologismo superfluo perché - si dice - esiste già una parola per dire la stessa cosa. No. Spesso due parole che hanno lo stesso referente non dicono affatto la stessa cosa, e cioè non sono omologhe. Per esempio, dire « ruolo» non è lo stesso che dire « ufficio » (anche se la parola ruolo può designare un ufficio); cosí come dire «struttura » non è lo stesso che dire « istituzione » (anche se la parola struttura può designare una istituzione). E quante volte azzecchiamo una risposta giusta azzeccando la parola giusta! Nomina numina. Dunque l'innovazione terminologica è da condannare come superflua solo quando non innova nella directio interpretationis. E se non si avverte questo, non si ha titolo di criticare.

In sintesi, la nuova politica comparata è tale perché affronta nuovi problemi, problemi che sembrano richiedere una renovatio $a b$ imis del nostro apparato concettuale ${ }^{19}$. In particolare, alla nuova politica comparata si pone il problema di acquisire concetti « capaci di viaggiare », categorie transcontinentali, per

${ }^{19}$ Occorre però distinguere tra coloro che innovano il vocabolario in funzione di nuovi approcci sistematici - ad esempio David Easton e Karl Deutsch - e coloro che cercano deliberatamente di ricostruire la terminologia. A quest'ultimo effetto l'autore piú lucido è Fred W. Riggs, di cui segnalo: The Comparison of Whole Political Systems, nel vol. cit., 
cosí dire. Questo è il problema di fondo. Ed è il problema che $\mathrm{mi}$ propongo di approfondire in questo saggio.

Vale avvertire subito che nel riconoscere l'esigenza di acquisire « categorie viaggianti » non accedo a quella varietà di caccia alle streghe che accusa i nostri concetti di essere culture-bound, viziati da etnocentrismo occidentale, e che ne ricava una sorta di dottrina del ripudio, e cioè il programma di sostituire i concetti etnocentrici con concetti «culturalmente incondizionati». A questo modo rischiamo di venire catapultati in una eterea terra di nessuno. E a me non sembra che dalla consapevolezza di un condizionamento culturale discenda alcuna necessità di fare un massacro e di ripartire da una tabula rasa. In primo luogo, posto che un concetto sia etnocentrico ne deriva soltanto che la sua applicabilità è da circoscrivere all'etbnos in questione. In secondo luogo, nulla vieta che dalla consapevolezza dei condizionamenti culturali venga desunta una dottrina del rispetto degli etnocentrismi altrui - e cioè il dovere di acquisirli senza snaturarli - e per essa un programma di internazionalizzazione poli-etnica del vocabolario politico. In terzo luogo, e in ogni caso, il problema di costruire dei concetti culturalmente incondizionati è solo un problema tra $\mathrm{i}$ tanti.

Ferma restando, allora, l'esigenza di una politica comparata globale, resta da vedere come l'impresa venga affrontata e perseguita. Dico subito che se l'esigenza è valida, il modo di viaggiare non convince. Salvo lodevoli eccezioni, i piú tendono a seguire la linea di minore resistenza, la via che dico dello stiramento dei concetti. La trovata è antica come il mondo: si aumenta la capienza di un concetto sfumandone la definizione. $\mathrm{Ma}$ è una trovata che mostra lestamente la corda: quel che si guadagna in capienza si perde in precisione. Per coprire piú terreno, finiamo per dire poco, e per dire poco in modo sempre meno preciso. Approdiamo cosí a concetti sempre piú vaporosi, piú amorfi, piú indefiniti. E questa non è davvero una soluzione. Anzi, è una soluzione cosí controproducente e ingenua che occorre farsene una ragione.

Formuliamo il problema. Non si nega che una politica comparata globale debba approdare a categorie o concetti « univer-

The Methodology of Comparative Research; Bureaucratic Politics in Comparative Perspective, in "Journal of Comparative Administration 》, I (1969), pp. 5-38; e Systems Theory: Structural Analysis, nel vol. coll. curato da M. Haas e H. Kariel, Approaches to the Study of Political Science, Scranton, Penn., Chandler, 1970. 
sali », validi per ogni luogo e tempo. Ma questi debbono essere universali empirici, e cioè tali da non sfuggire alla verifica, o falsificazione, empirica. L'universale filosofico - il concetto puro che Croce definiva «ultra-rappresentativo » - non ci serve: non ci serve, appunto, perché la sua purezza è supra-empirica. Ora, il problema dell'universale empirico è davvero irto di difficoltà. A tanto maggior ragione stupisce che non venga affrontato di petto. Perché? L'interrogativo può essere generalizzato in questa forma: come mai si presta una cosí inadeguata attenzione al come della comparazione, e cioè alla metodologia della comparazione?

Accenno rapidamente a due attenuanti, per soffermarmi poi sulla ragione di fondo. La prima attenuante è che, sinora, non si è ritenuto che al politologo occorresse un addestramento logico e metodologico. Il suo addestramento si limita, di solito, alle tecniche di ricerca e di manipolazione statistica. E il politologo - al pari del sociologo - non avverte questa lacuna per due ragioni. La prima è l'abuso della dizione «metodologia delle scienze sociali ». Abuso, perché nei trattati cosí intitolati manca, di solito, il proprio del metodo logico, e cioè manca il pensare sul pensare ${ }^{20}$. Mettiamola cosí: se la metodologia è padronanza della struttura e procedura logica del conoscere scientifico, questa padronanza fa difetto tanto al politologo quanto al sociologo. E gli interessati stentano a rendersene conto dicevo - anche per una seconda ragione, e cioè perché finora non erano mai stati costretti a procedere su un terreno vergine con il solo sostegno delle proprie gambe. Finora i cultori delle scienze sociali hanno vissuto a questo effetto di rendita, e cioè hanno implicitamente accettato che i propri problemi metodologici venissero risolti - o non risolti - da altri. E la sfida della comparazione globale che li costringe, volenti o nolenti, ad uscire allo scoperto. Ma non si abbandona d'un colpo una nicchia secolare. E quindi non deve sorprendere che le prime

${ }^{20}$ È il caso del noto William J. Goode e Paul K. Hatt, Methods in Social Research, trad. con il titolo Metodologia della ricerca sociale, Bologna, Il Mulino, 1962, che in metodologia è davvero debole, e che è, in verità, un testo di «tecnica » della ricerca. Cosí come è il caso dei testi cit. infra, nota 24. Peraltro la differenza tra metodi e tecniche è sempre piú avvertita. Ė sintomatico questo recente giudizio complessivo di A. Przeworski e $\mathrm{H}$. Teune: «molti libri ... sono cosí assorbiti dalla presentazione di specifiche procedure e tecniche da non discuterne nemmeno le giustificazioni e le implicazioni », (The Logic of Comparative Social Inquiry, New York, Wiley, 1970, p. X). 
sortite siano metodologicamente ingenue e incaute.

Una seconda attenuante fa capo, invece, al problema della Werfreibeit, della weberiana « libertà dal valore ». Senza entrare nel merito di questa controversia (anch'essa non risolta, o mal risolta), sta di fatto che la connotazione valutativa di un concetto ne costituisce spesso l'elemento qualificante. Pertanto il tentativo di neutralizzare i nostri concetti è sovente approdato, tout court, ad un loro svuotamento di significato: quel che si è guadagnato in avalutatività è stato pagato da una perdita di connotazione. Anche qui abbiamo dunque una « ricaduta nel generico », una vaporizzazione dei concetti. E prima di rivolgere il rimprovero ai comparatisti, equità vuole che si ricordi che essi hanno trovato la strada spianata. Se i comparatisti peccano di genericità per poter viaggiare, il loro peccato ha da gran tempo l'autorevole avallo di una Wertfreibeit probabilmente male intesa e certamente male applicata.

Se queste sono le attenuanti, qual è la ragione di fondo? Il mio quesito era come mai la politica comparata è ingenuamente caduta nella trappola dello « stiramento del concetto », invece di affrontare di petto il problema metodologico del come, di come comparare su scala globale. La risposta, o la giustificazione di maggior peso e momento, mi sembra essere questa: che non si tratta di perfezionare il « discorso qualitativo »; si tratta, invece, di sostituirlo con un « discorso quantitativo ». Non ci dobbiamo piú chiedere: cosa $\grave{e}$ ? $\mathrm{Ci}$ dobbiamo invece chiedere: quanto? In sostanza, la soluzione dei problemi pendenti è rinviata alla quantificazione, e in ultima analisi alla matematizzazione delle scienze sociali.

Piú che di una risposta si tratta, in fondo, di una proposta: la proposta di cambiare terreno, e quindi di giocare un nuovo gioco con nuove regole. Comunque sia, certo è che la « ricetta quantitativa » è creduta. $\mathrm{E}$ su questo punto occorre davvero intendersi ${ }^{21}$.

\section{Quantificazione e formazione dei concetti.}

La proposta quantificante e matematizzante viene argomentata, grosso modo, come segue. Di tanto i nostri concetti indicano differenze di genere (qualitative), e di tanto usiamo una

${ }^{21}$ I paragrafi che seguono sono, in larga misura, una rielaborazione del mio articolo Concept Misformation in Comparative Politics, in «American Political Science Review », LXIV (1970), pp. 1033-1053. 
logica dicotomica di identità-differenza, o di inclusione-esclusione, di altrettanto ci imbattiamo in difficoltà insuperabili. $\mathrm{Ma}$ se $i$ nostri concetti indicano differenze di grado (quantitative), e se usiamo una logica del piú-o-meno, allora le nostre difficoltà possono essere risolte dalla misurazione, e il vero problema diventa come misurare. In attesa, e cioè in attesa delle misure, $i$ « concetti di genere » (e le classificazioni) debbono essere accantonati, o quantomeno guardati con sospetto, dal momento che rappresentano « una vecchia logica di proprietà e attributi che mal si adatta allo studio delle quantità e delle relazioni ${ }^{22}$. La proposta è indubbiamente seducente, e indubbiamente aiuta a capire come mai i problemi metodologici prima posti e altri che andrò ponendo - siano stati cosí facilmente elusi o negletti. Ma non è una proposta che convince per la sua chiarezza.

Per veder chiaro occorre, in primo luogo, ripulire il terreno dall'abuso di un verbalismo quantitativo che è soltanto tale. E invalsa la moda di usare ad ogni pié sospinto le parole "grado » e « misurazione ». $\mathrm{Ma}$ - si è giustamente rilevato — il piú delle volte usiamo questi vocaboli « non soltanto senza disporre di alcuna misurazione effettiva, ma senza averne alcuna in progetto e, peggio ancora, senza nessuna apparente cognizione di quel che occorre fare prima che una misurazione diventi possibile ${ }^{23}$. Questo abuso idiomatico si è diffuso al punto da essere recepito persino nei testi tecnici, nei testi ad hoc: come quando troviamo che le cosiddette scale nominali vengono registrate come «scale di misurazione ${ }^{24}$. No, questo no: perché una scala nominale è soltanto una classificazione - una classificazione qualitativa - e dunque non è per niente una scala che misuri qualcosa. S'intende che anche le voci di una classificazione possono essere numerate: ma questo è solo un espediente di codifica che non ha nulla a che fare con una quantificazione.

22 Don Martindale, Sociological Theory and the Ideal Type, nel vol. curato da L. Gross, Symposium on Sociological Theory, New York, Harper \& Row, 1959. Questa frase riassume efficacemente la posizione di Carl Hempel.

${ }^{23}$ Abraham Kaplan, The Conduct of Inquiry, San Francisco, Chandler, 1964 , p. 213.

${ }^{24}$ Anche in testi eccellenti, quali Leon Festinger e Daniel Katz (eds.), Research Metbods in the Bebavioral Sciences, New York, Dryden Press, 1953, pp. 473-74; e Claire Selltiz, Jahoda, et al., Research Methods in So. cial Relations, ed. rev., New York, Holt, Rinehart \& Winston, 1962, pp. 189-90. 
Quel che si può concedere è, al massimo, che la misurazione comincia in pratica con le scale ordinali, anche se in teoria (e cioè ad effetto delle loro proprietà matematiche) le prime scale che davvero misurino sono le scale a intervallo ${ }^{25}$.

Analogamente, l'uso incessante della frase « è questione di grado », cosí come il frequentissimo ricorso alla immagine del continuum, tutto questo non ci avvicina di un solo millimetro alla quantificazione: ci lascia dove eravamo, e cioè ad un discorso qualitativo affidato a stime impressionistiche. Ancora, parliamo tutto il tempo di «variabili » che non sono tali, o che lo sono solo impropriamente, dal momento che non riguardano attributi graduabili e tantomeno attributi passibili di misurazione. Nulla di male finché diciamo variabile per civetteria, sapendo che potremmo anche dire « concetto ». Il male comincia quando non si afferra la differenza tra il modo di dire e il significato tecnico. $\mathrm{E}$ dunque sia chiaro: non basta dire « variabile » per averla.

Sgombrato il campo dal polverone sollevato dal verbalismo quantitativo, possiamo parlare di quantificazione sul serio, e cioè capire di cosa si tratti. Il confine tra l'abuso e il corretto uso del termine quantificazione è netto, e non è difficile da tracciare: la quantificazione comincia con i numeri, e quando i numeri sono usati per e con le loro proprietà aritmetiche. Semmai la difficoltà sta nell'orientarsi a valle di questo confine, e cioè nel seguire i molteplici possibili sviluppi della quantificazione. A questo fine conviene distinguere - a dispetto degli strettissimi nessi, e senza guardare troppo per il sottile - fra tre aree di applicazione, e cioè tra una quantificazione intesa come: 1) misurazione, 2) trattamento statistico, 3) formalizzazione matematica.

In scienza politica il grosso della quantificazione riguarda la prima accezione, e cioè una qualche forma di misurazione. Piú precisamente la quantificazione della scienza politica si risolve, il piú delle volte, in una di queste tre operazioni: a) l'attribuzione di valori numerici (misurazione pura e semplice), $b$ ) il rank ordering, cioè la determinazione di posizioni scalari (scale

${ }^{25}$ Per le considerazioni che autorizzano questo abbassamento della soglia alle scale ordinali, vedi Edward R. Tufte, Improving Data Analysis in Political Science, in «World Politics », XXI (1969), spec. p. 645. Si deve anche tener presente, ai fini di questa discussione, l'esistenza di scale intermedie, e spec. della scala metrica ordinata (intermedia tra scale ordinali e a intervallo). 
ordinali), c) la misurazione di distanze o intervalli (scale a intervallo).

$\mathrm{E}$ ben vero che a questa prima area di quantificazione si applicano, e si aggiungono, potenti tecniche di trattamento statistico; e non solo al fine di proteggerci da errori di campionamento e di misurazione, ma al fine di stabilire correlazioni e soprattutto relazioni significative tra variabili. Il balzo in avanti sta, appunto, nella scoperta di « relazioni ». Ma è proprio a questo punto che lo strumento statistico trova, in scienza politica, i suoi grossi limiti di applicazione. Per procedere statisticamente non ci occorrono tanto e soltanto dei grandi numeri; ci occorrono soprattutto variabili «rilevanti » che misurino le cose che ci interessa misurare, e variabili che misurino queste cose « validamente ». E queste due ultime condizioni sono difficili da soddisfare. Se riesaminiamo, difatti, le nostre cosiddette scoperte statistiche alla luce della loro rilevanza teoretica, ne risulta una sconfortante coincidenza tra bravura manipolativa e irrilevanza. Coincidenza che non è fortuita.

Quanto alla terza area di applicazione e accezione - la quantificazione che diventa una formalizzazione matematica lo stato della questione è che, sinora, tra scienza politica e matematica intercorre soltanto « una conversazione occasionale ${ }^{26}$. Il dialogo piú avanzato avviene sul terreno della teoria dei giochi, e di riflesso sul terreno della teoria delle coalizioni e delle decisioni ${ }^{27}$. L'esercizio è interessante e altamente stimolante. Senonché tanto le premesse quanto i risultati sono altamente irreali. Il fatto è che non riusciamo a stabilire corrispondenze isomorfiche tra relazioni empiriche fra cose, da un lato, e relazioni formali fra numeri, dall'altro lato.

Sarà magari una difficoltà pro tempore; e certo questa è la

${ }^{26}$ Oliver Benson, The Mathematical Approach to Political Science, in Contemporary Political Analysis, cit., p. 132. Il cap. di Benson contiene una buona rassegna della letteratura. Per un testo ad boc, vedi Hayward R. Alker, Matbematics and Politics, New York, Macmillan, 1965.

27 Per un primo orientamento e la bibliografia relativa, vedi, nel vol. cit. Contemporary Political Analysis, i ss. capp.: Thomas C. Schelling, What is Game Theory?; Martin Shubick, The Uses of Game Theory; Robinson and Majak, The Theory of Decision-Making; James N. Rosenau, The Premises and Promises of Decision-Making Analysis. Per la teoria delle coalizioni in particolare, cfr. William H. Riker, The Theory of Political Coalitions, New Haven, Yale Univ. Press, 1962; e S. Groennings, E. W. Kelley e M. Leiserson (eds.), The Study of Coalition Bebavior, New York, Holt, Rinehart and Winston, 1970. 
tesi di coloro che auspicano e prevedono la matematizzazione della scienza politica ${ }^{28}$. Ma guardiamo all'economia, e cioè alla piú quantitativa delle scienze sociali. Nel caso dello sviluppo matematico dell'economia la matematizzazione non ha preceduto, $\mathrm{ma}$ « ha sempre seguito i progressi qualitativi e concettuali $\gg{ }^{29}$. Si potrà eccepire che le analogie sono sempre insidiose, e in particolare che le matematiche di oggi hanno sopravanzato quelle di ieri, talché nulla vieta che la matematizzazione, da trainata che era mezzo secolo fa, diventi trainante. Si può convenire che di per sè non è detto che la sequenza che ha caratterizzato lo sviluppo dell'economia debba valere anche per le altre scienze sociali ${ }^{30}$. Il punto è se questa sequenza prima i concetti, poi la matematizzazione - abbia una sua ratio. Ragion d'essere che a me pare ci sia.

Quel che sfugge alla proposta matematizzante è il problema della formazione dei concetti. Noi pensiamo, o quantomeno veniamo addestrati a pensare, mediante un linguaggio qualitativo, un linguaggio naturale. $E$ non c'è modo di pretermettere il fatto che il nostro capire - il modo in cui funziona la mente umana - è costitutivamente condizionato, in partenza, dai «tagli » che corrispondono alla articolazione di un dato linguaggio naturale. Il nostro intelligere afferra il finito, non l'infinito, ciò che è diviso e articolato, non l'indiviso e l'indifferenziato. Né si può sostenere che questi «tagli », questi punti di divisione, possono essere ricavati statisticamente, e cioè lasciando che siano i dati a dirci dove stanno. Questa è davvero una tesi di corta vista nella quale un punto di arrivo oscura il punto di partenza: le «mappe concettuali » che stabiliscono

28 A questo effetto si punta sulle c.d. «matematiche dell'uomo », e cioè sulle matematiche non-quantitative, 0 anche discontinue (non sono la stessa cosa). Cfr. John G. Kemeny, Mathematics without Numbers, in Daniel Lerner (ed.), Quantity and Quality, Glencoe, Free Press, 1961; e J. C. Kemeny, J. L. Snell, G. L. Thompson, Introduction to Finite Mathematics, Englewood Cliffs, Prentice-Hall, 1957; trad. it. Matematica $e$ attività umane, Milano, Feltrinelli, 1968.

29 Joseph J. Spengler, Quantification in Economics: Its History, in Quantity and Quality, cit., p. 176. Spengler soggiunge che «l'introduzione in economia dei metodi quantitativi non ha prodotto scoperte sorprendenti $»$. Per quanto la teoria economica formale sia oggi altamente isomorfica con l'algebra, l'economia matematica non ha aggiunto molto al potere predittivo della disciplina, e si ha spesso l'impressione di un cannone che uccide una zanzara.

30 Per come la quantificazione entra nelle varie scienze sociali il vol. coll. cit. Quantity and Quality fornisce un eccellente panorama d'insieme. 
di che cosa il mondo è fatto. Talché prima di arrivare ai dati che parlano da soli occorre fare $\mathrm{i}$ conti con una articolazione fondamentale del linguaggio e del pensiero (del pensiero che è onomatologia) che viene costruita e ricostruita logicamente mediante l'affinamento concettuale della semantica dei linguaggi naturali - non certo da misurazioni. Misurazioni di che cosa? Non possiamo misurare se non sappiamo prima che cosa è che stiamo misurando. «Prima di poter accertare la presenza o meno di un attributo... o prima di poter graduare degli oggetti o di misurarli in ragione di una qualche variabile, dobbiamo formare il concetto di quella variabile ${ }^{31}$.

Dunque la formazione dei concetti sta prima della quantificazione (misurazione) e la condiziona. Ecco perché non ha molto senso costruire dei sistemi formalizzati di relazioni ben definite (cioè dei modelli matematici) finché vaghiamo in una nebbia di mal definiti concetti qualitativi. Ecco la ratio dell'ordine di sequenza che ha caratterizzato lo sviluppo dell'economia. Ed ecco perché è poco plausibile che le scienze sociali possano progredire quantitativamente $-\mathrm{e}$ in via quantitativa - senza aver prima raggiunto uno stadio soddisfacente di sistemazione concettuale.

Ripeto: la materia prima della quantificazione - le cose alle quali attacchiamo i numeri - non può essere fornita dal quantum, dal quantificare. Ne discende che le regole che presiedono alla formazione dei concetti non possono essere ricavate dalle regole che presiedono al trattamento delle quantità e dei rapporti quantitativi. $\mathrm{Ne}$ discende, cioè, che le regole che governano la formazione dei concetti sono indipendenti, e prioritarie, rispetto alle regole di altre fasi del procedimento euristico.

A detta della proposta matematizzante, ai concetti di genere dovrebbero sottentrare concetti di grado, talché dovremmo sorvolare sulla domanda « che cosa? », per insistere sempre

31 Paul F. Lazarsfeld e Allen H. Barton, Qualitative Measurement in the Social Sciences: Classifications, Typologies and Indices, in D. Lerner, H. D. Lasswell (eds.), The Policy Sciences, Stanford, Stanford Univ. Press, 1951, p. 155 (il corsivo è mio). Gli autori definiscono « variabile » cosí: « un attributo passibile di un qualsiasi numero di graduazioni, il quale implica, per di piú, la possibilità di essere misurato nel senso piú esatto della parola » (ibidem, p. 170). Questa definizione rigorosa può essere attenuata in ordine all'ultimo requisito; ma certo il significato tecnico di variabile non è applicabile - come ho già notato - agli attributi che non sono nemmeno graduabili. 
piú, e soltanto, sulla domanda « quanto? ${ }^{32}$. Ma non esiste un quantum che non sia di qualcosa. La questione pregiudiziale è sempre quanto in che cosa, e cioè in quale contenitore concettuale. Il che mi porta a concludere che la cosiddetta logica della gradazione (del piú-o-meno) è soltanto un elemento interno della logica del simile-dissimile, o dell'identità-differenza: che è poi la base della logica classificatoria.

\section{Classificazioni, dati e ricerca.}

La logica classificatoria e le classificazioni costituiscono oramai è chiaro - un motivo ritornante e un passaggio obbligato del mio argomento. Sarà bene, a questo punto, che io mi spieghi meglio. In primo luogo, una classificazione non è una mera enumerazione, un semplice elenco di voci. Per passare da una mera elencazione a una classificazione occorre un criterio: appunto, un criterio di classificazione ${ }^{33}$. In secondo luogo, una classificazione, o una tassonomia, sono tali a condizione che siano composte da classi complessivamente esaurienti e singolarmente esclusive. Sulla prima condizione si può essere tolleranti; ma la seconda condizione è tassativa. Le classi debbono essere reciprocamente esclusive, il che implica che i concetti di classe rappresentano caratteristiche che una fattispecie deve avere oppure non avere. Pertanto quando confrontiamo due oggetti, occorre innanzitutto stabilire se appartengono o non appartengono alla stessa classe, se possiedono o non possiedono uno stesso attributo. Se lo possiedono, e soltanto se lo possiedono, li possiamo confrontare in termini di piú o

32 In sede politica l'autore che persegue questo programma con maggiore finezza è Felix E. Oppenheim. Cfr. Dimensions of Freedom. An Analysis, New York, St. Martin's Press, 1962, capp. 8-9 e spec. pp. 179 . 80 (trad. it. Dimensioni della libertà, Feltrinelli, 1964). Nei vari scritti successivi questo leit-motiv si accentua. Il punto è, comunque, che la proposta matematizzante caratterizza, sia pure con molto minore consapevolezza teorica, la piú recente produzione dei giovani politologi nordamericani di osservanza behavioristica.

33 S'intende che possiamo combinare assieme piú di un criterio. In tal caso si usa dire tassonomia, o anche tipologia. Ai nostri fini questa distinzione non fa differenza. Su tutte queste questioni, lo scritto cit. di Lazarsfeld e Barton in The Policy Sciences resta tra $\mathrm{i}$ piú precisi e perspicui: vedilo ora nella raccolta curata da Vittorio Capecchi degli scritti di Lazarsfeld: Metodologia e ricerca sociologica, Bologna, Il Mulino, 1967, pp. 231-306. 
meno, e cioè possiamo passare ad accertare quale dei due oggetti abbia quell'attributo in misura maggiore o minore.

Dal che si vede che la gradazione è un elemento interno della classificazione. Prima occorrono le classi: poi, all'interno di ogni classe, intervengono le misurazioni. Passando da una classificazione ad una gradazione, passiamo dai segni egualediverso ai segni eguale-maggiore-minore. Pertanto l'identità posta dalla logica classificatoria resta la condizione di applicabilità dei segni piú-meno. È verissimo, dunque, che la gradazione completa e integra la classificazione. Ma è altrettanto vero che la gradazione presuppone la classificazione.

S'intende che non tutti $\mathrm{i}$ quantitativisti cadono nell'errore di rifiutare la logica classificatoria. Blalock, per esempio, sostiene una tesi piú blanda: «che mentre è sempre possibile, tecnicamente, pensare in termini di attributi e di dicotomie, il problema è se ciò sia pratico ${ }^{34}$. Si noti: Blalock dà per scontata la piena convertibilità del quantificare rispetto al classificare. Sul che basta che io ribadisca il mio dissenso. Piuttosto merita riprendere l'altro spunto: quanto sia « pratico $\gg$ procedere classificando.

In ultima analisi, e parlando in generale, l'esércizio classificatorio è un esercizio di «sbrogliamento » dei concetti. Procedere per genere e differenza equivale a spacchettare dei pacchetti concettuali, a sbrogliare dei gomitoli di idee. In sostanza, dunque, il classificatore è una tecnica di dispiegamento dei concetti. Non solo li decompone in una serie ordinata e maneggevole di voci, ma, decomponendoli, ne sviluppa le potenzialità. $\mathrm{E}$. siccome non disponiamo di tecniche di dispiegamento alternative, non vedo come si possa negare l'utilità pratica dell'esercizio classificatorio.

Ciò posto, veniamo ai problemi specifici della politica comparata. Ho già rilevato come la comparabilità presupponga una « omogeneità » stabilita procedendo per genus et differentiam. Due o piú cose sono paragonabili in quanto appartengono - per le caratteristiche che li rendono tali — ad una stessa

${ }^{34}$ Hubert M. Blalock Jr., Causal Inferences in Nonexperimental Research, Chapel Hill, University of North Carolina Press, 1964, p. 32. E interessante notare che in Social Statistics, New York, McGraw-Hill, 1960, cap. 2; trad. it. Statistica per la ricerca sociale, Bologna, Il Mulino, 1969, Blalock sosteneva una ben diversa tesi: posto che non riusciamo a misurare gran parte delle nostre variabili al punto da tradurle in scale a intervallo, può essere piú pratico trattare queste variabili come attributi. 
classe. Per converso due o piú cose eterogenee - che non appartengano allo stesso genere - non sono comparabili ${ }^{35}$. I quantitativisti non danno gran peso a questo aspetto del problema. Se esplicitato, il loro argomento sembra essere che gli studiosi si dedicano da gran tempo alla comparazione, e che hanno sempre mostrato di sapersi orientare a lume di naso, intuitivamente, senza troppo preoccuparsi di definire «il paragonabile ». E vero. Ma la situazione è cambiata per due importanti rispetti: la globalità della nuova politica comparata e, al contempo, l'entrata in scena degli elaboratori elettronici.

Dopo aver detto che lo studioso che restava confinato nell'area occidentale era culturalmente condizionato, resta da dire che, nell'esserlo, capiva, o capiva meglio, le cose di cui trattava. Il comparatista tradizionale viaggiava anche poco, d'accordo: ma là dove arrivava, arrivava davvero. Aggiungi che egli non disponeva di dati quantitativi, o comunque che non era un quantomane. Per tutte queste ragioni - o limitazioni - il comparatista pre-globale poggiava su un terreno relativamente solido, e cioè era controassicurato da un sapere personale, da una conoscenza di causa. Conoscenza sostantiva che si assottiglia quanto piú aumenta il numero dei paesi comparati, e che davvero si vanifica con l'avvento degli elaboratori e con un trattamento elettronico delle informazioni che si risolve, in larghissima misura, in un trattamento quantitativo.

Alcuni anni orsono Karl Deutsch prevedeva che per il 1975 la scienza politica avrebbe potuto contare su un magazzino di informazioni equivalente a circa 50 milioni di schede IBM, « con un tasso di crescita annuale di circa 5 milioni ${ }^{36}$. Milione piú, milione meno, la previsione dimostra sicuramente una cosa: che non ci potremo piú orientare a lume di naso. La rivoluzione elettronica è un fatto irreversibile e, ci piaccia o meno, rivoluzionerà anche il nostro modo di lavorare. $\mathrm{Ma}$ perciò rischiamo di essere inondati, anzi sopraffatti, da fiumane di cifre incontrollabili: incontrollabili alla luce di un vaglio sostantivo fatto da una mente umana. E se non vogliamo essere travolti, occorre provvedere in input, in entrata. In output, in uscita, non c'è piú sapere personale che tenga. Vale a dire, il

35 Vedi supra par. 2.

${ }^{36}$ Karl Deutsch, Recent Trends in Research Metbods, nel vol. coll. curato da J. C. Charlesworth, A Design for Political Science: Scope, Objectives and Methods, American Academy of Political and Social Science, Philadelphia, 1966, p. 156. 
problema è di disciplinare l'alimentazione dell'elaboratore. Hic Rbodus, bic salta.

I nostri predecessori avevano dunque una guida che noi abbiamo perduto, poiché oggi non possiamo piú fare affidamento sulla familiarità dello studioso con le cose che studia. E paradossale, pertanto, che i requisiti classificatori della comparabilità vengano negletti proprio nel momento in cui la globalizzazione della politica, e ancor piú la «computerizzazione » dei dati, ne richiede tassativamente l'esplicitazione. Per riprendere il quesito di Blalock, direi che lo spacchettamento dei concetti per genus et differentiam diventa una necessità « pratica » vitale anche e proprio al fine di disciplinare e di standardizzare l'informazione che alimenta gli elaboratori. Come si vedrà ancor meglio passando a considerare come questa informazione viene reperita.

Finora mi sono occupato del procedimento di formazione dei concetti, sostenendo che l'esercizio classificatorio costituisce un passaggio obbligato di tale procedimento. Il che può lasciar pensare che il mio interesse sia piú teoretico che empirico. Ma non è cosí, dal momento che i concetti delle scienze sociali non sono soltanto elementi di un sistema teoretico: sono egualmente, e contestualmente, dei contenitori di dati. I cosiddetti dati non sono altro che informazione distribuita in, e raffinata $d a$, « contenitori concettuali ». In questo senso teoria e ricerca sono due facce di una stessa medaglia. Ed è giunto il momento di approfondire questa seconda faccia, la faccia del fact-finding.

Dal momento che le scienze non-sperimentali si fondano su osservazioni esterne (non su osservazioni di laboratorio), e cioè su osservazioni di fatti, il problema empirico verte, in ultima analisi, su questa domanda: come convertire un concetto in un valido raccoglitore di fatti? Domanda alla quale si può rispondere che tanto minore è il potere discriminante di una categoria, tanto peggio viene raccolta l'informazione, e cioè tanto maggiore è la disinformazione. Viceversa, tanto maggiore il potere discriminante di un raccoglitore concettuale, tanto migliore l'informazione.

Si ribatterà che la risposta è vaga. Sí e no. Ė vaga se ne caviamo solo la raccomandazione che, in sede di ricerca, conviene semmai esagerare nel differenziare - nel reperire dati scorporati, minuti - piuttosto che nell'assimilare. Raccomandazione che, per quanto vaga, resta aurea: perché non si ripete- 
rà mai abbastanza che ci occorrono dati da aggregare, non « aggregati » già fatti. Inoltre la risposta non è affatto vaga se si tiene presente che il potere discriminante di una categoria non è affidato al libito del ricercatore, ma è stabilizzato - se lo vogliamo stabilire con un metro standardizzato - dall'ana. lisi per genere e differenza. Di tanto approfondiamo questa analisi, e cioè di tanto discendiamo - nello sfogliamento tassonomico - verso classi e sottoclassi sempre piú sottili, di altrettanto ne ricaviamo categorie sempre piú discriminanti. $\mathrm{E}$ dunque non solo disponiamo di una bussola per orientarsi al riguardo, ma disponiamo anche di un procedimento per convertire $\mathrm{i}$ concetti in strumenti di ricerca, e per ciò stesso in validi raccoglitori di dati.

Da quanto sopra risulta che l'esercizio classificatorio non è soltanto un momento costitutivo della formazione dei concetti; è anche, correlativamente, un momento costitutivo della ricerca. Difatti è proprio in sede di ricerca che possiamo riscontrare meglio gli inconvenienti, per non dire i danni, prodotti dalla moda quantitativizzante, e per essa dall'abbandono della logica classificatoria.

Da tempo i dipartimenti nordamericani di sociologia e di scienza politica inviano i propri candidati al dottorato di ricerca a giro per il mondo « in spedizioni indiscriminate di pesca di dati ${ }^{37}$. Queste spedizioni di pesca sono «indiscriminate » proprio perché difettano di reti adeguate; e le reti sono difettose perché non sono reti tassonomiche, e cioè per difetto di spacchettamento classificatorio. Questi ricercatori partono con il solo bagaglio di una checklist, di una lista di voci da spuntare, che equivale nel migliore dei casi ad una lacunosa rete da pesca privata. A questo modo il singolo ricercatore ha forse la vita piú facile. Ma per una disciplina che può crescere soltanto additivamente, e che abbisogna disperatamente di dati comparabili e cumulabili, $i$ frutti sono amari. Al tirar delle somme, l'impresa collettiva di una politica comparata globale è minacciata da un crescente potpourri di informazioni dispersive, disparate, poco addizionabili e probabilmente ingannevoli.

Si osserverà che $\mathrm{i}$ nostri dati non provengono soltanto

37 Cosí, spiritosamente, Joseph LaPalombara, Macrotbeories and Microapplications in Comparative Politics, in "Comparative Politics », I (1968), p. 66. 
dalle ricerche sul campo degli studiosi di scienze sociali. Vediamo meglio. Seguendo Karl Deutsch possiamo distinguere tra i seguenti settori di documentazione rilevanti, o di maggior promessa, per la scienza politica: 1) dati aggregati, e cioè $i$ dati dei censimenti, delle statistiche economiche, sociali, demografiche, e simili; 2) dati di opinione (ricavati dai sondaggi di opinione) e atteggiamento (ricavati da interviste); 3 ) dati elettorali e di votazioni (ivi includendo i comportamenti di voto legislativi); 4) dati sulle élites (estrazione, reclutamento e loro potere); 5) dati secondari o rigenerati mediante elaborazioni statistiche di data-making; 6) dati storici ${ }^{38}$.

La prima cosa che colpisce in questo elenco sono le lacune: lacune che rinviano, appunto, alla necessità delle spedizioni di ricerca sopra menzionate. In secondo luogo sorvolo sui dati volumetricamente minori, quali i dati storici (voce 6) e altri che non ho trascritto. In terzo luogo possiamo accantonare $i$ dati «generati da dati » (voce 5), visto che i dati rigenerati presuppongono pur sempre dei dati primari. Inoltre non occorre soffermarsi sui dati elettorali o di voto (voce 3 ) e sui dati di élites (voce 4), che sono i piú noti. Infine, per quanto riguarda la ingente massa dei dati di opinione (voce 2) basterà osservare che, nell'insieme, sono poco comparabili e di dubbia attendibilità. $\mathrm{Dal}$ che si ricava che - a parte gli studi elettorali, di voto e di élites - per tutto il resto finiamo inevitabilmente per far capo ai dati che Deutsch chiama aggregati (voce 1 ), e che sono dati statistici nel senso che sono rilevati dagli statistici. Sono questi, allora, i dati sui quali è bene intendersi ${ }^{39}$.

Dalla classe generalissima dei dati statistici vale scorporare i dati rilevati direttamente dagli economisti, o comunque da quella statistica ad boc che è la statistica economica. Sono,

38 Cfr. Deutsch, A Design for Political Science, cit., pp. 152-157, anche per la bibliografia relativa. Complessivamente Deutsch distingue nove settori. Per una esemplificazione di quanto si può fare, in sede di analisi politica, sulla base delle rilevazioni esistenti (nel paese meglio fornito di dati) si può vedere J. M. e W. C. Mitchell, The Changing Politics of American Life, in E. B. Sheldon, W. E. Moore (eds.), Indicators of Social Change Concepts and Measurements, New York, Russell Sage Foundation, 1968, pp. 247-294.

39 Per orientarsi in concreto, cfr. Russett et al., World Handbooie of Political and Sociale Indicators, New Haven, Yale University Press, 1964, nuova ed. 1969. Questo testo è la fonte piú comprensiva dei dati che hanno una qualche rilevanza per le scienze sociali. Vi si guardi, in particolare, la sezione Government and Politics. 
questi, i dati di gran lunga piú soddisfacenti. Ma vanno scorporati per due ragioni. La prima è che servono $\mathrm{i}$ diversi fini di un diverso utente. La seconda ragione è meno ovvia, ma piú importante: è che il sociologo e il politologo non possono commissionare il reperimento dei propri dati alla stessa stregua e con la stessa facilità dell'economista. Tantovero che la statistica economica è ben diversa dalla cosiddetta statistica sociale. La differenza è che soltanto l'economista fruisce di valori quantitativi che esprimono, anzi incarnano, i comportamenti stessi degli osservati (visto che gli attori economici sono guidati da criteri quantitativi); laddove $i$ valori quantitativi sui quali lavorano le altre scienze dell'uomo sono « ricostruzioni » $\mathrm{e}$ 《 attribuzioni » dell'osservatore. La differenza è capitale. $\mathrm{E}$ con questo siamo finalmente arrivati alla documentazione che ci interessa da vicino: le statistiche genericamente dette economico-sociali, le cui variabili di rito sono, all'incirca, la scolarizzazione e l'istruzione, le occupazioni e le professioni, la distribuzione sociale del reddito, l'urbanizzazione e l'industrializzazione. Documentazione che naviga - lo dico senza perifrasi in pessime acque.

Per quanto si dica che la statistica è uno strumento aperto, a disposizione di tutti i suoi possibili utenti, la verità è che gli statistici servono determinati utenti e non altri, a cominciare da se stessi (anche sub specie di demografi). Non è il caso di entrare nella diatriba delle recriminazioni reciproche tra statistici e studiosi di scienze sociali. Lo stato dei fatti è che $\mathrm{i}$ criteri e le classi di rilevazione degli istituti di statistica (nazionali o meno) sono generalmente antiquati, grossolani, e internazionalmente non standardizzati ${ }^{40}$. Ne consegue, in primo luogo, che quando combiniamo le statistiche dei singoli paesi siamo subito colpiti dal sospetto che le stesse voci (quando sono le stesse) non misurino affatto gli stessi fenomeni ${ }^{41}$. Sospetto destinato a restare tale perché gli aggregati sono quelli che sono: poco o punto discriminanti. Poco discriminanti agli

40 Non alludo in particolare all'ISTAT. Cfr. per es. le severe critiche alle rilevazioni statistiche negli Stati Uniti di Bertram Gross, The State of the Nation - Social Systems Accounting, nel vol. coll. curato da R. A. Bauer, Social Indicators, Cambridge, M.I.T. Press, 1966.

${ }^{41}$ Per le difficoltà di paragonabilità cfr. Richard Merritt, Stein Rokkan (eds.), Comparing Nations; The Use of Quantitative Data in CrossNational Research, New Haven, Yale University Press, 1966. Peraltro gli autori sottovalutano le difficoltà in questione. 
stessi fini della statistica comparata; punto discriminanti ai fini delle analisi sociologiche e politologiche ${ }^{42}$. Con questo non si chiede che gli statistici si mettano al servizio delle scienze sociali $^{43}$. Si chiede però che utilizzino davvero gli elaboratori di cui oggi dispongono, e che forniscano « dati disaggregati » multi-purpose - e cioè utilizzabili ai fini di una pluralità di utenti - in luogo di «dati aggregati » che inevitabilmente sono aggregati ai fini di un solo utente, e quindi risultano singlepurpose, valevoli per una sola destinazione. Detto questo, gli statistici hanno a loro volta ragione $\mathrm{da}$ vendere quando lamentano che sono gli utenti - in questo caso $\mathrm{i}$ sociologi e $\mathrm{i}$ politologi - che non precisano la loro domanda. Su questo non posso che convenire, visto che il loro lamento è il mio stesso lamento.

Dicevo che l'intrapresa collettiva di una politica comparata globale è insidiata da un guazzabuglio di informazioni eterogenee, poco addizionabili e probabilmente ingannevoli. Come si è veduto, questa diagnosi non muta, anzi viene rinforzata, se mettiamo in conto, oltre ai dati di ricerca, la documentazione statistica corrente. Aggiungo, e preciso, che in ordine a questa diagnosi non $\mathrm{fa}$ differenza che l'informazione sia qualitativa o quantitativa. In ogni caso la terapia è la stessa: costruire «categorie di ricerca » munite di un adeguato potere discriminante. Se i nostri contenitori di dati sono concettualmente indefiniti e confusi, ne risulteranno dati ambigui e mistificanti, dati che pasticciano assieme il simile e il diverso. Semmai la differenza sta qui: che i danni prodotti da una « disinformazione quantitativa » sono - rispetto a quella qualitativa - maggiori e piú insidiosi. Incalcolabilmente maggiori grazie alla computerizzazione; e infinitamente piú insidiosi per-

42 Valga il solo esempio delle statistiche sull'istruzione, in base alle quali si cercano, tra l'altro, correlazioni tra alti livelli di istruzione e sviluppo democratico. Correlazioni che non hanno senso se, e finché, l'istruzione è rilevata soltanto in termini di anni di scolarizzazione. Gli anni non qualificano, da soli, un «alto livello». E lo stesso numero di anni può essere destinato 1) ad un indottrinamento democratico, 2) ad un indottrinamento anti-democratico, 3) ad una istruzione puramente tecnologica.

${ }_{43}$ E peraltro lecito chiedere che nella misura in cui uno stato destina le sue risorse alla "politica sociale », nella stessa misura commissioni ai propri servizi statistici il reperimento di adeguate informazioni sociali. Il che non è davvero il caso dell'ISTAT, talché tutta la nostra politica sociale naviga nel piú profondo buio. 
ché una disinformazione quantitativa su scala globale sfugge al vaglio del nostro sapere sostantivo e finisce per essere utilizzata senza alcuna conoscenza di causa.

Vale concludere. $\mathrm{Ma}$, prima, ricapitoliamo tutto il discorso dei due ultimi paragrafi. Ho sostenuto che la logica di identitàdifferenza, o di inclusione-esclusione, non può essere sostituita dai segni piú-o-meno: si tratta, invece, di due sintassi logiche complementari, e che si integrano nell'ordine che va dalla prima alla seconda. Correlativamente ho sostenuto che il ripudio delle classificazioni ha gravi ripercussioni negative, e risulta del tutto ingiustificato. Hempel concede che $\mathrm{i}$ concetti di classe si prestano alla descrizione delle osservazioni e alla prima formulazione di approssimative generalizzazioni empiriche ${ }^{44}$. Ma gli sfugge che l'esercizio classificatorio gioca un ruolo insostituibile nella formazione dei concetti, poiché ne costituisce la tecnica di «spacchettamento ». Senza contare che le classificazioni introducono una preziosa chiarezza e articolazione analitica nel discorso, dal momento che ci inducono a procedere con ordine, discutendo una cosa alla volta e cose diverse in volte diverse. Al che si deve aggiungere che abbiamo assoluto bisogno di reti classificatorie e di reticolati tassonomici al fine di risolvere i nostri problemi di fact-finding e di fact-storing, di ricerca e di immagazzinamento dei dati.

In conclusione, nessuna scienza politica comparata è plausibile, su scala globale, in difetto di estese informazioni che siano abbastanza precise da consentire un controllo comparato valido e significativo. A questo fine ci occorre, in via pregiudiziale, un sistema di schedatura che sia molto articolato, relativamente stabile, e per ciò stesso cumulabile ai fini dell'incremento e dell'aggiornamento dei dati. Ma il paradosso è che tanto piú ci orientiamo verso il trattamento elettronico dell'informazione, e tanto meno siamo in grado di supplire informazioni raccolte con criteri logici standardizzati. Talché l'età dell'informativa rischia di capovolgersi nell'età delle pseudo-informazioni.

Alle fonti di questo mal passo sta - a mio giudizio una ingiustificata svalutazione della logica Aristotelica delle categorie e l'indebito perfezionismo epistemologico e metodolo-

${ }^{44}$ Cfr. Carl G. Hempel, Fundamentals of Concept Formation in Empirical Science, Chicago, Univ. Chicago Press, 1952, p. 54 (trad. it. La formazione dei concetti e delle teorie nella scienza empirica, Milano, Feltrinelli, 1961). 
gico di chi, come Hempel, riconosce un solo modello di « scienza »: quello delle scienze fisico-sperimentali. A questo modo veniamo incitati a correre con gambe sbagliate, e in ogni caso con gambe che sono appena sufficienti per camminare. Pondere et mensura di che cosa? Nelle scienze sperimentali - una volta costituite come tali - è l'esperimento stesso che isola e circoscrive il « che cosa ». Nelle cosiddette scienze formalizzate, o nelle matematiche, il che cosa è irrilevante. Ma nelle scienze osservative il « che cosa » sta prima di tutto. Sta tuttora prima di tutto in quelle scienze osservative solide e consolidate che sono la mineralogia, la botanica e la zoologia. A tanto maggior ragione il «che cosa » non può che stare prima di tutto nelle scienze osservative ancora fluide, e del fluido: le scienze dell'uomo. Talché il consiglio hempeliano e matematizzante di guardare alle quantità e alle relazioni diffidando dell'analisi per genere e differenza, per proprietà ed attributi, si risolse in un vero e proprio usteron pròteron, nel mettere il carro davanti ai buoi. A questo modo rischiamo di ripudiare una «scienza di specie » in cambio di niente.

Le conseguenze di questi errori a monte sono visibilissime a valle. Si riscontrano, in primo luogo, in una attività di ricerca altamente dispersiva e triviale: tale perché né guidata né sorretta da congrui « raccoglitori concettuali ». Ed emergono an-cora piú clamorosamente quando guardiamo allo stato della documentazione. Lo stato dei dati è caotico; e lo sarà sempre di piú, se non ci convinceremo di quanto l'analisi e la standardizzazione classificatoria siano necessarie. Senza l'ausilio di raffinati reticolati tassonomici, e senza un correlativo sistema di schedatura stabilizzato, non illudiamoci: gli elaboratori ci faranno piú male che bene ${ }^{45}$. L'informatica è preziosa; a patto che non diventi l'oppio degli studiosi.

La scala di astrazione.

Ripartiamo da questa considerazione: la nuova politica comparata ha imboccato, alternativamente o congiuntamente, due vie senza sbocco: da un lato uno «stiramento del concetto » che ci riporta alla notte hegeliana nella quale tutte le vacche

${ }^{45}$ Un eloquente, pessimo precedente di compilazione affidata alle macchine è Arthur S. Banks e Robert Textor, A Cross-Polity Survey, Cambridge, M.I.T. Press, 1963. 
sembrano nere; dall'altro una «misurazione senza concetto » che ci lascia piú deboli e sprovveduti che mai. Due vie senza sbocco, sí; ma a fronte di due esigenze valide: acquisire universali empirici capaci di viaggiare, e arrivare a variabili misurabili. Qual è, allora, la via da battere?

Il problema resta un problema di formazione dei concetti. E la prospettiva nella quale ora mi pongo è quella della disposizione verticale degli elementi di una struttura concettuale lungo una scala di astrazione.

Premetto due avvertenze. La prima è che dico «concetto » per brevità, e cioè con l'intesa che riferisco all'elemento concettuale anche una serie di elementi che in una trattazione piú estesa e meglio articolata appartengono, in verità, alla voce « proposizioni ». Piú esattamente, nel dire formazione del concetto alludo, implicitamente, ad una attività formatrice di proposizioni e risolutrice di problemi. La seconda avvertenza è che il mio discorso verte, implicitamente, su una particolare classe di concetti: quelli espressi da termini osservativi piuttosto che da costrutti teorici. Va anche da sé, correlativamente, che non mi interesso a tutti i concetti di questa classe, ma solo ai concetti-chiave della disciplina: concetti che sovente coincidono con le « generalizzazioni camuffate » di Bendix ${ }^{46}$.

La nozione di scala di astrazione è collegata alla esistenza di diversi livelli di analisi; ma un alto livello di astrazione non risulta necessariamente da un processo di ladder climbing, di «scalata astraente », e cioè di salita lungo una scala di astrazione. Il che equivale a dire che una serie di universali non è ricavata, non è « astratta », da cose osservabili. In tal caso abbiamo a che fare, appunto, con dei costrutti teorici, o termini teoretici, definiti dalla loro collocazione nel sistema concettuale al quale appartengono ${ }^{47}$.'Per esempio, il significato di

46 Supra nota 1.

47 Seguo Kaplan, The Conduct of Inquiry, cit., pp. 56-57, 63-65, adottandone la terminologia. Secondo Hempel, i termini teoretici non rilevano, di solito, « ad entità direttamente osservabili e alle loro caratteristiche ... Attengono ... alle teorie scientifiche intese a spiegare generalizzazioni » (in Minnesota Studies in the Pbilosopby of Science, di Feigl, Scriven, Maxwell (eds.), Minneapolis, Univ. of Minnesota Press, 1958, vol. II, p. 42). La distinzione tra termini teoretici e termini osservativi non è ontologica, è segnata da confini mobili (molti termini sono di dubbia attribuzione, se non promiscui), e il solo punto fermo sulla questione è l'irriducibilità dei primi ai secondi. Per un recente riepilogo di questo dibattito, cfr. Alberto Meotti, L'eliminazione dei termini teorici, in 
termini quali isomorfismo, omeostasi, feedback, entropia e simili, è definito, in ultima analisi, dal ruolo che assumono all'interno della teoria generale dei sistemi. In altri casi, invece, arriviamo a alti livelli di astrazione mediante una scalata astraente, salendo lungo una scala di astrazione. In tal caso abbiamo a che fare con termini osservativi, vale a dire con termini ricavati da cose osservabili, o meglio ricavati tramite inferenze astraenti che fanno capo, in qualche modo, a osservazioni dirette $o$ indirette. Per esempio, termini quali gruppo, comunicazione, conflitto, decisione e simili, possono essere intesi in modo concretissimo (in riferimento a gruppi reali, comunicazioni emesse o ricevute in proprio, conflitti e decisioni che avvengono qui e ora), oppure possono essere usati in un significato altamente rarefatto, cioè astratto (malamente detto, dai politologi, « analitico »); ma anche nel secondo caso resta vero che si tratta di termini riconducibili in qualche misura a eventi e cose osservabili. In questo senso, e in antitesi ai costrutti teorici, $i$ termini osservativi possono anche essere chiamati concetti empirici. Nel qual caso si dirà che i concetti empirici sono tali perché riferibili od osservabili, anche se un concetto empirico può essere collocato a livelli di astrazione ben diversi, e anzi è caratterizzato dal fatto di muoversi lungo una scala di astrazione.

Pertanto il nostro problema si formula cosí: 1) stabilire a quale livello di astrazione vogliamo collocare i concetti empirico-osservativi, e 2) conoscere le regole di trasformazione relative, e cioè le regole di percorrimento di una scala di astrazione. Difatti il problema di fondo della politica comparata è di conseguire guadagni in estensione, $\mathrm{o}$ in capienza (salendo lungo la scala astraente), senza subire perdite innecessarie, o comunque irrecuperabili, in termini di precisione e di controllabilità.

Per venire a capo di questo problema occorre cominciare dal fermare bene la distinzione-relazione tra estensione (o denotazione), e intensione (o connotazione) di un termine. Una definizione standard è questa: «L'estensione di una parola è la classe di cose alle quali la parola si applica; l'intensione di

«Rivista di Filosofia », LX (1969), n. 2. La distinzione è da me accolta al solo fine di mettere in evidenza che $\mathrm{i}$ termini teorici non sono ricavabili induttivamente, per «scalata astraente »: possono essere sviluppati solo deduttivamente. Per converso i termini osservativi sono caratterizzati da uno sviluppo induttivo. 
una parola è l'insieme delle proprietà che stabiliscono a quali cose la parola è applicabile ${ }^{48}$. Analogamente, per denotazione si intende la «totalità degli oggetti », o eventi, alla quale la parola si applica; laddove per connotazione si intende la «totalità delle caratteristiche » che qualcosa deve possedere per rientrare nella denotazione di quella parola ${ }^{49}$.

Ciò posto, è subito facile capire qual è il modo corretto di salire una scala di astrazione. La regola è semplice: per aumentare l'estensione di un termine se ne deve ridurre la connotazione. Cosí procedendo ricaviamo, di volta in volta, un termine piú «generale », o piú inclusivo, che non diventa per questo un termine impreciso. E chiaro che tanto maggiore è la capienza di un concetto, tanto minori sono le differenze - proprietà o attributi - che ne vengono captate: ma quel potere di differenziazione che gli resta, resta tal quale, cioè mantiene la precisione che aveva. Non è tutto. Cosí procedendo ricaviamo anche concettualizzazioni le quali, per quanto onnicapienti, possono sempre essere ricondotte - facendo il cammino a ritroso, e cioè ridiscendendo la scala di astrazione - a «specifici » passibili di verifica o falsificazione empirica.

Con questo abbiamo anche identificato la natura dell'errore nel quale incappano i comparativisti nella loro fretta di acquisire universali capaci di viaggiare, oltre l'urbe, per tutto l'orbe. Lo «stiramento del concetto » non è altro che il tentativo di aumentare l'estensione dei concetti senza diminuirne l'intensione: talché la denotazione viene estesa offuscando la connotazione. Con il risultato di ottenere non concetti piú generali, ma la loro contraffazione, e cioè mere generalità, o meglio ancora mere genericità. La differenza è che un con. cetto generale (che sussume una molteplicità di specie entro un piú largo genere) prelude a «generalizzazioni » scientifiche, laddove delle mere generalità, dei concetti « generici », approdano soltanto a discorsi vaghi e confusi.

Le regole per salire, o per scendere, una scala di astrazione sono dunque regole assai semplici, almeno in linea di principio.

48 Cosí Wesley C. Salmon, Logic, Englewood Cliffs, Prentice-Hall, 1963, pp. 90-91 (trad. it. Logica elementare, Bologna, Il Mulino, 1969). $\mathrm{Ma}$ è una definizione che appare, con poche varianti, in molti testi di logica.

49 "Connotazione » è anche usato in un significato piú lato, per le associazioni di idee evocate dall'uso di un termine. E inteso, pertanto, che qui uso connotazione nel suo significato piú stretto, come omologo di «intensione ». 
Rendiamo un concetto piú astratto e piú generale riducendone le proprietà o attributi. Viceversa, un concetto viene reso piú specifico dall'aggiunta o dispiegamento di qualificazioni, e cioè aumentandone le proprietà $o$ attributi. E queste sono non solo le regole di trasformazione dei concetti empirico-osservativi, ma anche le regole di costruzione di una scala di astrazione. Ciò posto, vediamo di puntualizzarne lo schema.

E di per sé evidente che lungo una scala di astrazione si possono collocare moltissimi livelli di inclusività e, viceversa, di specificità. Ai fini di una schematizzazione basterà distinguere, comunque, tre fasce o zone altimetriche; 1) alto livello di astrazione (AL), 2) medio livello di astrazione (ML), 3) basso livello di astrazione (BL). Sono concetti $\mathrm{AL}$, di alto livello, le categorie universali applicabili ad ogni luogo (geograficamente) o tempo (storicamente): in questo caso la connotazione è drasticamente sacrificata al requisito di una denotazione globale o onnitemporale. Nella fascia dei concetti ML, di medio livello, troviamo invece categorie generali (ma non universali): in questo caso l'estensione viene bilanciata con l'intensione, anche se l'esigenza è di "generalizzare », e quindi di rilevare le similarità a scapito delle differenze. Infine, sono concetti $\mathrm{BL}$, di basso livello, le categorie specifiche che si svolgono in concezioni dette configurative (forse traducibili con il termine ideografiche) $\mathrm{e}$ in definizioni « contestuali »: in questo caso la denotazione viene sottoposta al requisito di una connotazione accurata (individualizzante), talché sono le differenze che prevalgono sulle somiglianze.

Vale spiegarsi con un esempio. In un saggio che affronta i problemi dell'economia comparata (che non sono, concettualmente, diversi da quelli della politica comparata) lo Smelser osserva che ai fini di una comparazione globale «staff è meglio che amministrazione ... e amministrazione è meglio che civil service ». A detta di Smelser, infatti, la nozione di civil service non è applicabile ai paesi che non possiedono un apparato statale strutturato; la nozione di amministrazione è relativamente «superiore, ma resta culturalmente condizionata »; talché staff si residua ad essere il termine « atto a coprire senza difficoltà i piú vari arrangiamenti politici ${ }^{50}$. Accettando per buone queste

${ }^{50}$ Neil J. Smelser, Notes on the Methodology of Comparative Analysis of Economic Activity, in Transactions of the Sixth World Congress of Sociology, Evian, International Sociological Association, 1966, vol. II, p. 103. 
proposte terminologiche, nei miei termini l'argomento di Smelser sarebbe da svolgere come segue. Nel contesto della pubblica amministrazione comparata, la categoria universale è staff. Il concetto di amministrazione ha difatti una buona applicabilità generale, ma non universale, per via delle associazioni che lo legano all'idea di burocrazia. Ancora piú limitata è la denotazione di civil service, qualificata dagli attributi dello Stato moderno. Se poi vogliamo scendere la scala sino al basso livello di astrazione, un esame comparato del civil service, mettiamo, inglese e francese rivela profonde differenze e richiede definizioni contestuali. Vale aggiungere che nell'esempio in questione il discorso è semplificato dall'esistenza di una gamma di vocaboli che ci consente (quale che sia la scelta) di identificare ogni livello di astrazione, o quasi, con una denominazione a sé. $\mathrm{Ma}$ vi sono casi piú sfortunati nei quali, per difetto di vocabolario, siamo addirittura costretti a percorrere tutta la scala di astrazione con uno stesso termine.

Va da sé che, in concreto, i livelli di astrazione non sono necessariamente tre, e sono spesso assai piú di tre. Il numero delle fette dipende da quanto le vogliamo rendere sottili, e cioè dalla meticolosità di una analisi. E altrettanto ovvio che le varie fette di una struttura concettuale verticale non sono necessariamente divise da precisi confini. Molti passaggi verticali sono davvero sfumati e graduali. Pertanto se il mio schema lascia pensare a due confini, e se li riferisce a tre, e soltanto tre, livelli di astrazione, è perché questo ritaglio sembra sufficiente e significativo ai fini di una analisi logica. Quel che mi interessa è dunque la «logica» delle operazioni che avvengono lungo una scala di astrazione. A questo effetto il problema piú spinoso è quello del movimento ascendente; vale a dire un problema che si colloca alla giuntura che divide i concetti generali (ML) dalle categorie universali ( $A L)$, e che si formula cosí: fino a che punto possiamo far salire un termine osservativo senza che soccomba a un eccesso di « sforzo astraente »?

In linea di principio, una classe non dovrebbe essere allargata oltre il punto in cui verrebbe a perdere anche l'ultima sua connotazione (proprietà o attributo) precisabile. $\mathrm{Ma}$ a questo modo si chiede molto: perché si chiede una identificazione positiva. In pratica, alle categorie universali finiamo per chiedere molto meno: soltanto una identificazione negativa, a contrario. Sta bene. Ma ancor meno di cosí non sta piú bene. 
Vale pertanto fermare la distinzione capitale tra: 1) concetti qualificati ex adverso, cioè dichiarando cosa non sono, 2) concetti senza contrario, e quindi senza termine. Questa distinzione risale al noto principio per cui omnis determinatio est negatio. Principio dal quale discende che un universale provvisto di contrario è sempre un concetto determinato, laddove un universale senza negazione diventa un concetto indeterminato. $\mathrm{E}$ questa distinzione logica è di fondamentale importanza empirica. Un universale determinato a contrario è pur sempre un concetto di cui si può affermare o negare l'applicabilità al mondo reale; laddove un universale indeterminato si applica per definizione: non avendo termine, o delimitazione, non abbiamo modo di accertarne l'applicabilità o meno al mondo reale. E questa è, appunto, la differenza tra universali empirici e universali che non sono tali, e che dunque sono, dal punto di vista di una conoscenza empirica, pseudo-universali. Un universale empirico è tale perché sta ancora per « qualcosa »; laddove l'indeterminatezza dell'universale non-empirico richiama indiscriminatamente « ogni cosa ».

Una illustrazione calzante è fornita dalla cosiddetta «teoria del gruppo », e per essa da un concetto di gruppo che si pone come l'unità primaria di tutta la fenomenologia politica ${ }^{51}$. L'esempio è calzante anche perché la nuova politica comparata esordisce su scala mondiale proprio in questa chiave, e cioè traducendo la teoria del gruppo nelle ricerche sui "gruppi di pressione ${ }^{52}$. Nella group theory of politics « gruppo » è chiaramente una categoria universale. Il gruppo è la chiave di tutto, e tutto è gruppo. Senonché, non ci viene mai detto che cosa non è gruppo. Non solo questo concetto si applica ovunque, come si richiede ad un universale; ma si applica a tutto, e cioè non troveremo mai, da nessuna parte, dei non-gruppi, qualcosa che è

${ }^{51}$ Gli esponenti piú noti di questa dottrina, che fa capo all'opera di Arthur F. Bentley, The Process of Government (del 1908), sono Earl Lathan e specialmente David Truman, con The Governmental Process (del 1951). Per la bibliografia vedi, infra, note 52 e 53 .

${ }_{52}$ Per il manifesto di questa conversione vedi Gabriel Almond, $A$ Comparative Study of Interest Groups and the Political Process, in «A. merican Political Science Review », LII (1958), pp. 270-282. In generale cfr. Eckstein, Introduction: Group Theory and the Comparative Study of Pressure Groups, nell'antologia cit., Comparative Politics. A Reader, pp. 389-397; e Fisichella, Partiti politici e gruppi di pressione: Introduzione, in Antologia di scienza politica, cit., spec. pp. 271-276. 
meno o piú di un gruppo ${ }^{53}$. Secondo i criteri di cui sopra, allora, « gruppo » non è un universale empirico. Difatti quando andiamo a vedere le ricerche sui gruppi di interesse, o di pressione, è facile riscontrare che queste ricerche non sono orientate dal « gruppo indeterminato » della teoria, ma dal « gruppo intuitivo », e cioè da idee intuitive desunte dall'osservazione dei gruppi concreti. Nel migliore dei casi teoria e ricerca sono andate ognuna per proprio conto. Nel peggiore dei casi la teorizzazione ha smantellato quel che la ricerca andava scoprendo. $\mathrm{E}$ in ogni caso restiamo con una letteratura che acchiappa tutto e nulla, e cioè gravemente indebolita dalla insufficienza di nervatura teoretica, e particolarmente da un insufficiente inquadramento tassonomico ${ }^{54}$. Talché non fa meraviglia che alla euforia iniziale sia seguita la frustrazione, e che oggi la grande caccia globale ai gruppi di interesse sia stata pressoché abbandonata.

In conclusione, lo sforzo astraente verso una inclusività universale trova un suo punto di rottura oltre il quale sta soltanto una nullificazione del problema, o quantomeno una vaporizzazione empirica. Questo punto di rottura è segnato dal venir meno della stessa determinazione ex adverso. In tal caso abbiamo un universale empiricamente inutilizzabile. Con questo non dico inutile, o sprovvisto di senso. Quel che intendo dire è che dalla trasformazione di concetti come gruppo - o come pluralismo, integrazione, partecipazione e mobilitazione ${ }^{55}$ - in universali «senza termine » ricaviamo soltanto delle targhe, dei cartellini. Targhe e cartellini che non sono inutili perché servono ad indicare l'argomento, o anche una messa a fuoco; ma che non sono piú, in alcun modo, uno strumento di lavoro. A quest'ultimo effetto non solo il gioco non vale la candela, ma rischia di risolversi in un autoinganno.

Passiamo, o meglio scendiamo, dall'alto livello di astrazione

53 David Truman, che pure è il meno sfuggente degli autori in questione, esordisce, caratteristicamente, affermando «che una preoccupazione eccessiva per le definizioni è controproducente $»$ (op. cit., p. 23). Per un approfondimento di queste riserve vedi Giovanni Sartori, Gruppi di interese o gruppi di pressione? Una discussione sul neo-pluralismo, in «Il Mulino», VIII (1959), pp. 7-42. Ancor piú critico è Donald A. Strickland, Is there a Group Theory of Politics?, in «Il Politico », XXXV (1970), pp. 269-285.

54 Basti pensare alla mancata individuazione del fenomeno sindacale, sottinteso e mai fermato dalla rete concettuale dei gruppi di interesse.

55 Questi quattro concetti sono gli esempi analizzati nel mio articolo Concept Misformation in Comparative Politics, loc. cit. 
a quello medio. La fascia media, o intermedia, dei concetti « generali » dovrebbe essere una fascia molto spessa. Dovrebbe, dico, perché sta di fatto che spessa non è; e non lo è perché corrisponde a quel livello di astrazione - oggi atrofizzato nel quale dovremmo dispiegare e articolare i concetti per genus et differentiam. David Apter ha ragione di lamentare che «le nostre categorie analitiche sono troppo generali quando sono teoretiche, e troppo descrittive quando non lo sono ${ }^{56}$. Il suo lamento coglie il vuoto che esiste tra osservazioni descrittive e categorie universali, e per esso la natura acrobatica dei nostri salti tra basso e alto livello di astrazione. $\mathrm{E}$ per colmare questo vuoto, per passare davvero dalle osservazioni alle generalizzazioni - e viceversa - occorre sviluppare il medio livello di astrazione; il che equivale a dire che occorre tornare all'esercizio classificatorio, allo spacchettamento tassonomico. Non mi soffermo, perché è un discorso già fatto. Discorso dal quale si ricava che il medio livello di astrazione è nettamente caratterizzato da un suo modus operandi, da una « logica » che lo differenzia, appunto, dai livelli sovrastanti e sottostanti. Vale aggiungere che la fascia intermedia dei generi, delle specie e delle sottospecie, è la struttura portante di tutta una scala di astrazione. Il grosso del lavoro avviene qui, come è facile capire.

Resta il basso livello di astrazione, che potrebbe sembrare un livello di scarso interesse per il comparatista. Ma non è cosí. Se - dicevo - il problema piú spinoso si pone, almeno allo stato dei fatti, in sede di movimento ascendente, ciò non toglie che esista anche un problema di movimento discendente. Problema che si colloca, stavolta, alla giuntura che divide $\mathbf{i}$ concetti generali (ML) dalle concezioni « contestuali » (BL). Anche il comparatista è tenuto a fare ricerche, e anzi le deve fare per procurarsi i dati che gli occorrono. Ora è vero che la ricerca del comparatista non deve essere individualizzante né fine a se stessa; e quindi è vero che al comparatista si chiede di scendere sul campo avendo alle spalle una armatura concettuale generalizzante. Ma resta il fatto che le ricerche si fanno qui e ora, e che il proprio della ricerca consiste di osservazioni dirette $o$ indirette. $E$ dunque anche per il comparatista si pone il problema di scendere dal medio al basso livello di astrazione.

56 David Apter, Political Studies and the Search for a Framework, di prossima pubblicazione in C. Allen e W. Johnson (eds.), African Perspectives, Cambridge, Cambridge University Press. 
Altrimenti il comparatista rischia piú di ogni altro di scendere sul campo munito di « paraocchi deformanti » che precostituiscono e adulterano la registrazione dei fatti. Per minimizzare questo rischio occorrono categorie altamente discriminanti. Il che equivale a dire che quanto piú il comparatista saprà scendere verso, e calarsi dentro, il basso livello di astrazione, tanto meglio saprà osservare e cercare.

Finora l'esposizione è stata piú deduttiva che induttiva, nel senso che la costruzione della scala di astrazione non prende l'avvio - nel mio argomento - dal livello piú basso. Ma una volta che ci siamo arrivati, è bene rifare il cammino induttivamente. Anche perché a questo modo l'importanza del basso livello di astrazione viene in tutta evidenza.

Dicevo che $\mathrm{i}$ reticolati tassonomici sviluppati al medio livello di astrazione sono la chiave di volta di tutto l'edificio. Resta da dire che se una classificazione viene ricavata da regole logiche, la logica non ha nulla a che vedere con l'utilità e validità di una classificazione. I botanici e gli zoologi non hanno imposto le loro classi su piante o animali, piú di quanto piante e animali non si siano imposte ai loro classificatori. Vale a dire, le classificazioni valgono nella misura in cui superano la prova della ricerca, e cioè superano, in ultima analisi, il collaudo induttivo. Di per sé un edificio tassonomico è soltanto un insieme di cassetti vuoti: cassetti di cui non sappiamo, a priori, se si prestano o meno ad incamerare $i$ fatti. Lo possiamo scoprire solo induttivamente, e cioè nel momento in cui occorre trasferire una descrizione ideografica e contestuale - e cioè a basso livello di astrazione - nelle classi e, correlativamente, nei procedimenti astraenti e generalizzanti del medio livello di astrazione.

Vale riassumere l'argomento complessivo - lo schema della scala di astrazione - in una tabella, tabella che giova commentare.

Una prima osservazione è che non basta - al fine di contrassegnare un livello di analisi - predicare di un termine che lo usiamo in senso stretto oppure in senso lato. A fronte di una scala di astrazione, «stretto » $\mathrm{e}$ 《lato » non indicano se intendiamo distinguere tra: 1) universali $\mathrm{AL}$ e concetti generali $\mathrm{ML}$; 2) generi e specie del livello medio; 3) classi ML e specifici BL; 4) addirittura tra universali AL e configurazioni BL. Ovviamente non occorre essere sempre pignoli. $\mathrm{Ma}$ occorre esserlo 


\section{SCHEMA DELLA SCALA DI ASTRAZIONE}

$\begin{array}{ccc}\text { Livelli } & \begin{array}{c}\text { Scopo e ambito } \\ \text { di astrazione }\end{array} & \begin{array}{c}\text { Proprietà empiriche } \\ \text { e logiche }\end{array}\end{array}$

AL: Alto Livello:

Comparazioni inter-area Estensione massima (contesti eterogenei) Intensione minima

Concetti universali Teoria globale

Definizione a contrario

ML: Medio Livello:

BL: Basso Livello:

Comparazioni intra-area Equilibrio tra (contesti omogenei) denotazione-connotazione

Concetti generali Teoria a medio raggio

Concetti di classe

(tassonomie)

Definizione per genere

e differenza

(ricorrendo magari ad esponenti numerici) quando nel discorso si intrecciano molteplici sensi piú o meno stretti rispetto ad altrettanti sensi piú o meno lati.

L'osservazione importante è comunque questa: che la scala di astrazione porta in tutta evidenza la frivolità del detto: « tutte le differenze sono una questione di grado ». Questo metaforeggiare quantitativizzante si risolve in una drastica perdita di articolazione logica, e sottintende una sequela di errori che possiamo oramai seguire passo passo. In primo luogo è chiaro che 
all'alto livello di astrazione il problema è la rilevanza e determinatezza teorica del concetto. E altrettanto chiaro che nel tratto superiore del medio livello di astrazione le determinazioni iniziali sono necessariamente determinazioni di genere. Dopodiché cominciamo a scendere la scala con la tecnica del dispiegamento tassonomico, il che equivale a dire che ancora per un tratto il problema non è di gradi, ma piuttosto di specie. Si ricordi: le differenze diventano di grado solo dopo aver stabilito che due o piú oggetti hanno le stesse proprietà o attributi. E queste proprietà e attributi vengono isolate, di solito, al livello delle classi di specie, non a quello delle classi di genere. Pertanto il quesito di quali componenti di una classe abbiano le stesse proprietà in misura maggiore o minore è, il piú delle volte, un quesito che si sviluppa al livello che potremmo dire medio-basso.

L'errore è, dunque, in linea di principio, di ignorare la disposizione verticale dei concetti. Ma se ricordiamo che i concetti hanno una organizzazione verticale, e che per aumentare l'estensione di un termine ne dobbiamo ridurre la connotazione (e viceversa), ne consegue che finché manovriamo - in salita cosí come in discesa - lungo una scala di astrazione, la questione è se determinate proprietà $o$ attributi siano presenti oppure assenti: e questo non è un problema di gradi ma di identificare il livello di astrazione. È solo dopo, dopo aver stabilito a quale livello di astrazione siamo collocati, che intervengono le considerazioni di piú-o-meno. E la regola di massima sembra essere che tanto piú elevato è il livello di astrazione, tanto meno si applica l'ottica dei gradi; laddove tanto piú basso è il livello di astrazione, e tanto piú l'ottica dei gradi diventa pertinente e le misurazioni necessarie.

Una terza osservazione, generalissima, riguarda la tesi che affiora spesso nella letteratura metodologica, secondo la quale « tanto piú una proposizione è universale, e cioè tanto maggiore è il numero di eventi che ne vengono considerati, di altrettanto aumentano le possibilità di falsificazione e tanto piú informativa è la proposizione ${ }^{57}$. L'idea espressa da questa tesi è, in sostanza, che tra universalità, falsificabilità e contenuto informativo esista una progressione concomitante, talché il progresso

57 Cito da Erik Allardt, The Merger of American and European Traditions of Sociological Research: Contextual Analysis, in «Social Science Information », VII (1968), p. 165. 
di un elemento è anche, automaticamente, un progresso degli altri. Ma alla luce della scala di astrazione risulta una diversa conclusione: che ad ogni punto della scala dobbiamo scegliere tra raggio esplicativo e accuratezza descrittiva, tra quel che si guadagna in capienza e quel che si perde in dettaglio. Pertanto ci dobbiamo intendere su quel che Allardt chiama « contenuto informativo » di una proposizione. Una proposizione piú generale, o piú astratta, spiega di piú: ma descrive meno, e in questo senso informa meno. Dal che consegue che non c'è concomitanza necessaria tra maggiore astrazione e maggiore falsificabilità. Senza contare che, volendo troppo salire, finiamo anche per approdare ad universali che non sono piú falsificabili.

\section{Funzione e struttura.}

Fin qui il discorso metodologico a monte - sulla formazione-malformazione del concetto - che è il discorso che, di solito, non si $\mathrm{fa}^{58}$. Resterebbe da fare il discorso metodologico a valle, che è poi il discorso che si fa: e dunque un discorso che può essere rinviato alla letteratura ${ }^{59}$. Piuttosto conviene calare il mio argomento nel fatto, e cioè svolgerlo in chiave di esempi. Qui l'imbarazzo è davvero nella scelta. $\mathrm{Ma}$ i concetti di « struttura » e «funzione » - la mia scelta — si raccomandano per un duplice rispetto: non solo perché appartengono al novero

58 Tra le pochissime eccezioni vedi spec. i primi due capitoli del recentissimo The Methodology of Comparative Research, cit., rispettivamente di Holt e Turner e di Holt e Richardson (pp. 1-71). Questi autori svolgono peraltro il discorso metodologico a monte in tutt'altra direzione, rifacendosi a criteri di «deduzione paradigmatica» di cui non condivido il perfezionismo. Il volume a piú alto livello di approfondimento metodologico, per le scienze sociali in generale, è quello curato da Llewellyn Gross, Symposium on Sociological Theory: Inquiries and Paradigms, cit.

59 Beninteso, sempre la letteratura metodologica, non quella « tecnologica ». In primissimo luogo segnalerei il vol. curato da Paul F. Lazarsfeld e Morris Rosenberg, The Language of Social Research. A Reader, Glencoe, Free Press, 1955; ora riedito e rielaborato in due voll. da Raymond Boudon e Paul F. Lazarsfeld, Méthodes de la sociologie, Paris, Mouton, 1965-66, tradotto (omettendo «metodi » quando ci stava bene) con il titolo L'analisi empirica nelle scienze sociali, Bologna, Il Mulino, 1969. Segnalazione dalla quale già traspare la mia preferenza per Lazarsfeld, che mi induce a richiamare in questa sede il suo vol. già cit., Metodologia e Ricerca Sociologica. Per il settore specifico della comparazione collocherei in questo gruppo A. Przeworski e H. Teune, The Logic of Comparative Social Inquiry, cit. 
dei macro-concetti di frequente uso ed abuso; ma soprattutto perché fondano, al tempo stesso, un approccio: l'analisi strutturale-funzionale ${ }^{60}$.

Nel presentare il volume che piú di ogni altro ha dato l'avvio alla nuova politica comparata, Almond ne riassume l'impostazione cosí: "Quel che abbiamo fatto è dividere funzione politica da struttura politica ${ }^{61}$. Questo è davvero il problema. $\mathrm{Ma}$ tra il dire e il riuscire il passo è lungo. Sono passati dieci anni, e ancora il passo non è riuscito. Tantovero che siamo ancora invischiati nella questione pregiudiziale di cosa si debba intendere per «funzione », sia prendendo il termine a sé, sia nel suo rapporto con «struttura ».

Beninteso, in questa sede la nozione di funzione non interessa di per sé, ma per come sta rispetto a quella di struttura ${ }^{62}$. Per cosí dire, il concetto di funzione entra nel mio discorso « in funzione di ». Nondimeno le matasse debbono essere sbrogliate cominciando da un capo, e conviene cominciare da quello di «funzione». Per un matematico, quando l'elemento $y$ varia con l'elemento $x, y$ è una funzione di $x$ : e dunque funzione è soltanto una relazione. Ma noi diciamo che la funzione di una certa struttura $\grave{e}$, per dire: questa struttura $b a$ questa funzione. Ov. viamente quest'ultima frase non è da prendere alla lettera, e non si svolge nella tesi che le funzioni sono «cose possedute » dalle strutture. Per esteso l'argomento si svolge cosí: che le

60 William Flanigan e Edwin Fogelman, Functional Analysis, nel vol. cit. Contemporary Political Analysis, distinguono tra 1) funzionalismo eclettico, 2) funzionalismo empirico (Merton), 3) analisi strutturale-funzionale (pp. 72-79). Preciso subito che il mio discorso si applica solo all'analisi strutturale-funzionale, e, in questa, al solo contesto della scienza politica.

${ }^{61}$ Almond, The Politics of the Developing Areas, cit., p. 59. Sull'opera di Almond, che è l'autore di maggior spicco per l'analisi strutturale-funzionale, vedi l'introduzione e bibliografia di Gianfranco Pasquino, Per una teoria politica empirica alla trad. it. del vol. di Gabriel A. Almond e G. Bingham Powell, Politica comparata, Bologna, Il Mulino, 1970, pp. 7-34. Cfr. anche Giuliano Urbani, L'analisi del sistema politico, Bologna, Il Mulino, 1971, dedicato, per una buona metà, ad Almond.

${ }_{62}$ Tra l'altro, «funzione » sembra essere un costrutto teorico. L'esempio prescelto è dunque interessante non solo ad effetto della mobilità delle frontiere tra termini teorici e termini osservativi (supra nota 47), ma anche al fine di vedere - seguendo Carnap - le « regole di corrispondenza » che consentono di dare ai termini teorici quantomeno una « interpretazione parziale »: fermo restando che le osservazioni non danno al termine teorico il suo significato, ma che ne indicano le occasioni di applicabilità. 
strutture vengono in essere per fare qualcosa; che taluni aspetti ritenuti essenziali di quel «fare » vengono qualificati funzioni; dal che consegue che le funzioni sono attribuzioni (dell'osservatore) intese a caratterizzare la ragion d'essere delle strutture.

Fermiamo subito due punti. Il primo è che - salvo errori di ingenua reificazione - non c'è errore, ma anzi molto senso, nel dire che le strutture banno delle funzioni. Il secondo punto è che non basta dire che le funzioni sono attività delle strutture. Lo sono, beninteso: ma i partiti, le burocrazie, le chiese, gli eserciti, le legislature, i governi, e altre strutture ancora, dispiegano mille attività - anche importanti - che non sono considerate funzioni (e nemmeno disfunzioni). Dunque, se le funzioni sono attività, non tutte le attività sono funzioni. Né vale, per aggirare l'ostacolo, definire le funzioni come conseguenze, come effett $i^{63}$. Gli effetti sono poi « effetti di attività ». E l'obiezione resta che molte attività delle strutture hanno effetti, ed effetti rilevanti, senza che per questo ci venga in mente di registrarle come funzioni ${ }^{64}$.

Lo stato della controversia nella piú recente dottrina è dunque, in sintesi, che «funzione » viene variamente definita come: 1) relazione, 2) attività, 3) effetto ${ }^{65}$. Senonché nessuna di queste definizioni sembra afferrare davvero l'idea. Nessuno dubita che funzione sia un costrutto relazionale; ma l'accezione matematica non ci aiuta a calare la funzione nella struttura. Ánalogamente nessuno dubita che le funzioni siano attività; e possiamo concedere che tali attività sono da registrare in sede di conseguenze o effetti: ma quali attività, e perché taluni effetti

${ }^{63}$ Quest'ultima accezione risale a Robert K. Merton, la cui preoccupazione era di separare funzione - definita come «conseguenza oggettiva osservabile »- da « disposizioni soggettive », e cioè fini, motivi e obiettivi (Social Theory and Social Structure, Glencoe, Free Press, 1957, trad. it. Teoria e struttura sociale, vol. I: Teoria sociologica e ricerca empirica, Bologna, Il Mulino, 1971, p. 129, e passim pp. 121-224). L'accezione mertoniana è dunque giustificata da una preoccupazione di « osservabilità » intesa in chiave behavioristica e oggettivistica. $\mathrm{Ma}$ è altrettanto vero che questa messa a fuoco cerca una delimitazione rispetto alla onnicomprensività di « attività ».

${ }_{64}$ A conferma di questa obiezione cfr. Robert T. Holt, A Proposed Structural-Functional Framework, in Contemporary Political Analysis, cit., spec. 88-90, la cui sottile analisi, che riprende e sviluppa l'accezione mertoniana, approda a un circolo vizioso che ne dimostra l'inconclusività.

65 Per un approfondimento vedi spec. l'antologia a cura di Nicholas J. Demerath, Richard A. Peterson, System, Change and Conflict, New York, Free Press, 1967, dedicata in larga parte al dibattito sul funzionalismo. 
e non altri? Conveniamone: il problema non è semplice. E per venirne a capo occorre differenziare $i$ contesti, accettando l'idea che il termine funzione viene legittimamente usato, a seconda dei contesti, con significati diversi.

Ai nostri fini i contesti da distinguere sono tre: 1) la partial system analysis, e cioè l'analisi delle strutture (o sottosistemi) singolarmente considerate; 2) la whole system analysis, o analisi dei «sistemi interi »; 3) la systems analysis che deriva, o si riallaccia, alla cosiddetta teoria generale dei sistemi, alla general systems theory ${ }^{66}$. E la distinzione da fermare è soprattutto quella tra analisi general systems (voce 3) e analisi whole system (voce 2 ). Questione che è davvero pasticciata ${ }^{67}$.

Nel contesto della systems analysis quel che interessa non è l'attività delle strutture, ma le interazioni tra tutti gli elementi del sistema considerato. In questa prospettiva non diciamo che le strutture hanno funzioni; diciamo invece che un sistema (politico) è posto da elementi in equilibrio reciproco (dinamico) che variano l'uno in funzione dell'altro. Il che equivale a dire che il sistema è un insieme di interrelazioni funzionali: relazioni reciproche che - sapendolo fare - potrebbero essere espresse $\mathrm{da}$ equazioni. E chiaro, dunque, che nel contesto della systems analysis «funzione » è usata nell'accezione matematica del termine: funzione sta per « relazione ». Tutto bene, salvo che qui l'analisi strutturale-funzionale non c'entra: e non c'entra perché le strutture non ci sono, o meglio sono le $x$ e le $y$ dell'equazione.

Nella whole system analysis quel che interessa è sí, ancora, il sistema intero; ma il discorso è diverso ${ }^{68}$. Tanto diverso che

${ }^{66} \mathrm{Su}$ quest'ultima vedi, in via preliminare, Oran $\mathrm{R}$. Young, The Impact of General Systems Theory on Political Science, in "General Systems », IX (1964).

67 Pasticciata, in primo luogo, in dottrina. Cfr., anche per le discordanze, Morton Kaplan, Systems Theory, e Herbert J. Spiro, An Evaluation of Systems Theory, entrambi in Contemporary Political Analysis, cit.; Anatol Rapoport, Some System Approaches to Political Theory, e David Easton, Categories for the Systems Analysis of Politics, entrambi nel volume curato da Easton, Varieties of Political Theory, Englewood Cliffs, Prentice-Hall, 1966. Per intendersi in concreto, e in prima approssimazione, diciamo che Easton si ascrive alla analisi sistemica (come è anche messo in evidenza dal titolo del suo volume di maggior impegno: $A$ Systems Analysis of Political Life, New York, Wiley, 1965), laddove Almond appartiene alla analisi whole system. Le differenze tra $\mathrm{i}$ due costituiscono il motivo centrale del vol. cit. di Urbani, L'analisi del sistema politico.

${ }^{68}$ L'autore che persegue questa impostazione con maggior consape- 
in questo contesto si parla di «funzioni del sistema », e cioè vengono attribuite al sistema (all'insieme delle strutture che compongono il sistema politico) delle funzioni che sono tali nello stesso senso nel quale parliamo (nell'analisi parziale, o segmentata) di «funzioni delle strutture ». E vero che l'analista del sistema intero si interessa anche alle interazioni tra strutture. Ma questa analogia con la systems analysis non deve trarre in inganno, e cioè non deve far pensare che anche nel caso in esame le funzioni siano « relazioni ». No: le funzioni del sistema sono «attività » (pur essendo concepibile che queste attività funzionali siano il prodotto di interrelazioni funzionali), e piú precisamente quelle attività che sono rilevanti per il mantenimento, adattamento, o anche trasformazione, del sistema politico. Dal che mi sembra discendere che, a dispetto delle apparenze e delle polemiche intestine ${ }^{69}$, la whole system analysis è molto piú prossima all'analisi parziale che a quella general systems. Conclusione rafforzata da questa considerazione: che il sistema intero è pur sempre visto come un insieme interagente di «strutture » (non di incognite). Se cosí non è, lo studio del sistema intero fuoriesce dall'ambito dello strutturalfunzionalismo. Ma se cosí è - o nella misura in cui è cosí la conclusione diventa che il sistema intero presuppone il sistema segmentato, e cioè la conoscenza effettiva delle strutture che sono in questione. Dopotutto quel che l'analisi whole system sa delle strutture che ricombina assieme, lo sa da chi si dedica allo studio delle strutture singolarmente prese.

Veniamo cosí alla partial system analysis, all'analisi parziale o segmentata dei sotto-sistemi del sistema complessive. $\mathrm{E}$ veniamo direttamente al punto: cosa si intende, in questo contesto, per funzione. Non è un mistero di difficile decifrazione. Quel che lo rende tale è soprattutto una malposta preoccupazione avalutativa. Se noi giriamo - in sede di indagine struttural-funzionale - in tondo e a vuoto, è soprattutto perché timorosi di ammettere che il nostro è un discorso di Zweckrationalität, come direbbe Max Weber, e cioè di razionalità e ra-

volezza è F. W. Riggs. Cfr. spec. The Comparison of Whole Political Systems, e Systems Theory: Structural Analysis, entrambi richiamati supra, nota 19.

${ }^{69}$ Cfr. la critica alla analisi intera e la difesa di quella parziale di LaPalombara, Parsimony and Empiricism in Comparative Politics: An Anti-Scholastic View, in The Methodology of Comparative Research, cit. 
zionalizzazione dei fini ${ }^{70}$. Eppure non c'è dubbio. Noi intendiamo che le strutture esistono per: per qualche destinazione, compito o scopo. Quale che ne sia la definizione ufficiale, e a dispetto di ogni camuffamento terminologico, la sostanza è che funzione è, qui, un concetto teleologico che investe una relazione tra mezzi e fini. Piú esattamente, funzione è l'attività di una struttura - il mezzo - a fronte dei suoi fini. Questi fini possono essere intesi descrittivamente, e cioè risultare dalla dinamica endogena della struttura considerata e investire soltanto le mansioni effettivamente espletate; ovvero possono essere intesi prescrittivamente, alla luce dei cosiddetti fini istituzionali, o comunque dei fini che una struttura « dovrebbe » perseguire $^{71}$. Ma in ogni caso l'attività di una struttura è commisurata ad uno scopo, ad una destinazione: altrimenti non è una attività-funzione, ma una attività qualsiasi. Correlativamente, dicendo disfunzione, non funzionalità, e simili, intendiamo che i fini in questione non sono soddisfatti.

Fin qui «funzione » riferita alle singole strutture, e cioè nel contesto della partial system analysis. Ma si è già visto che l'analisi segmentata costituisce anche il sostrato della whole system analysis: quantomeno nella misura in cui quest'ultima vuol essere strutturale, e non soltanto funzionale ${ }^{72}$. Cosí come si è già visto che le «funzioni del sistema » non sono tali in una accezione irriducibilmente diversa da quella che vale per $\mathrm{i}$ sotto-sistemi. Se possiamo attribuire funzioni ai sotto-sistemi, alla stessa stregua e nello stesso senso possiamo attribuire fun-

${ }^{70} \mathrm{La}$ preoccupazione avalutativa è dunque malposta perché una $Z$ weckrationalität non è una Wertrationalität, una razionalità valutativa. Non lo è, tra l'altro, perché in sede di razionalità dei fini possiamo postulare l'equi-valenza, e cioè l'egual valore, di tutti i fini: talché possiamo recepire tutte le funzioni ipotizzabili, buone o cattive che siano.

71 Le cosiddette «funzioni inintenzionali », non previste e forse nemmeno volute, possono essere assorbite come una sottoclasse delle funzioni descrittivamente intese, e cioè effettivamente espletate. Quanto alle "funzioni latenti», esse pongono un problema solo a chi le vorrebbe registrare in sede di effetti.

${ }_{72}$ Un esempio di funzioni sistemiche ricavate da, e chiaramente riconducibili $a$, strutture sub-sistemiche è dato dalle tre originarie «funzioni di input» di Almond - rule making, rule application, e rule adjudication - che sono trasparentemente riferibili alle strutture governativo-legislative, amministrative e giudiziarie (The Politics of the Developing Areas, cit., p. 17). Queste tre funzioni sono ora riclassificate nel novero delle «funzioni di conversione» (cfr. Almond e Powell, Politica comparata, cit., spec. pp. 51 e 69). 
zioni al sistema sovraordinato. La differenza non è di concetto ma - a proposito - di livelli di astrazione (talché il problema di collegare il livello di analisi sub-sistemico a quello sistemico è riconducibile a un problema di salita astraente lungo una scala di astrazione). Le funzioni sistemiche sono sí funzioni « sintetiche » altamente astratte: ma restano attività mediate da strutture - i mezzi - e commisurate a scopi. E dunque il discorso funzionale a livello sistemico è analogo a quello subsistemico: resta un discorso di Zweckrationalität.

Il prologo è stato, di necessità, lungo. Ma ora - passando alle strutture - possiamo procedere spediti. Perché le difficoltà con «struttura » sono un riflesso di quanto già detto. Posto che la funzione è la ragion d'essere delle strutture, ne consegue che il modo piú agevole di identificare una struttura è di qualificarla in nome della sua ratio essendi. Ecco allora la difficoltà: che la maggior parte delle strutture politiche sono identificate o da una denominazione funzionale, o comunque da una definizione funzionale. Per il primo rispetto il nostro vocabolario funzionale (teleologico) è molto piú ricco del nostro vocabolario strutturale (descrittivo). E per il secondo rispetto le strutture non vengono quasi mai definite nei termini dovuti, e cioè come strutture. Per quanto si chieda, di una struttura politica, « cosa è », finiamo invariabilmente per rispondere in termini di «a cosa serve »: e dunque per tacere sul come $\grave{e}$, surrogandolo con una spiegazione sul perché c'è.

Cosa è una elezione? Un modo per eleggere. Cosa è una legislatura? Una assemblea per produrre leggi. Cosa è un governo? Un arrangiamento per governare. Cosa sono i partiti? Strumenti per far eleggere. E cosí via. Elezioni, legislature, governi, partiti, ecc. sono strutture: ma non è facile, né tantomeno sbrigativo, caratterizzarle come tali. E dunque comprensibile che le strutture vengano percepite e qualificate alla luce delle loro funzioni preeminenti ${ }^{73}$. Anzi, per il modo in cui $\mathrm{i}$ piú fanno politica va benissimo cosí. Ma va malissimo per chi studia la politica, e ancor peggio per chi attende alla ingegneria politica. In concreto, le riforme si fanno sulle strutture: e se non siamo in grado di stabilire con sufficiente precisione a

73 Sia chiaro: una caratterizzazione strutturale che sia veramente tale rinvia, e presuppone, una meticolosa «descrizione strutturale » che è necessariamente lunga (infra nota 76) e anche difficile da sintetizzare. Per contro le caratterizzazioni funzionali sono brevi e di maggior soddisfazione: invece di descrivere, spiegano. 
quali strutture corrispondono quali effetti (funzionali), l'ingegneria politica si trova davvero a mal partito ${ }^{74}$.

Almond ha ben ragione quando fonda il suo approccio sulla esigenza di separare struttura da funzione. Cosí come egli indica esattamente l'importanza dell'approccio per la politica comparata: «tutti i sistemi politici possono essere comparati in termini di relazione tra funzioni e strutture ${ }^{75}$. Ma Almond sottovaluta, e di parecchio, le difficoltà. Perché sta di fatto che lo struttural-funzionalista non cammina, a tutt'oggi, su due gambe, ma su una gamba sola: e, per di piú, su una gamba barlaccia. Fuor di metafora, il fatto è che egli non lavora su due termini che sono davvero due - la struttura per come riopera sulla funzione - ma piuttosto su strutture che restano inestricabilmente impigliate nelle loro attribuzioni funzionali. Donde un giro vizioso.

Basti pensare - per avvertire questo giro vizioso - alle tre conclusioni alle quali ogni struttural-funzionalista che si rispetti sembra accedere: 1) che nessuna struttura è uni-funzionale, e cioè che nessuna struttura assolve una sola funzione; 2) che la stessa struttura può essere multifunzionale, nel senso che può espletare, da paese a paese, funzioni ben diverse; 3) talché la stessa funzione trova alternative strutturali, e cioè può essere espletata da diverse strutture. Queste tesi sono senz'altro plausibili. $\mathrm{Ma}$ non erano tesi da scoprire: sapevamo già, a naso, che era cosí. Erano piuttosto tesi da determinare: perché non sapevamo fino a che punto fosse cosí. Interviene l'analisi strutturale-funzionale e, invece di determinarle, le generalizza, e semmai le assolutizza: tutto è fungibile. La struttura non vincola a nessuna funzione, e viceversa le funzioni non sono legate a nessuna struttura.

Il paradosso è che se la tesi multifunzionale fosse vera sarebbe suicida, e cioè dimostrerebbe che l'analisi strutturale è quantomeno superflua. Difatti se una stessa struttura funziona in modo tutt'affatto diverso da paese a paese, e se per ogni funzione vi sono alternative strutturali, a che pro occuparsi e preoccuparsi di strutture? Ma è davvero la stessa struttura

${ }^{74}$ Per uno svolgimento in chiave di ingegneria politica debbo rinviare al mio saggio Political Development and Political Engineering, in Public Policy, Cambridge, Mass., Harvard Univ. Press, 1968 (vol. XVII), spec. pp. 261-276 e 297-298.

75 Almond e Powell, Politica comparata, cit., p. 69. 
che funziona diversamente? Oppure il funzionamento è diverso perché - guardando meglio - la struttura non è la stessa?

Si prenda il caso delle elezioni. Le elezioni possono anche servire - lo sappiamo benissimo - a legittimare un despota. Ma non ne consegue che le elezioni siano «multifunzionali ». Per lo strutturalista le elezioni sono una struttura, e sono da precisare sub specie di strutture al plurale, di strutture che risultano diversissime. Per esempio le « elezioni libere » non sono strutturate come le elezioni illibere (quelle che plebiscitano e legittimano i dispotismi). La struttura delle elezioni libere richiede, tra l'altro, libertà di propaganda e di espressione, almeno una alternativa tra cui scegliere, effettiva segretezza del voto, nonché tutti quegli accorgimenti atti ad impedire brogli elettorali e un conteggio fraudolentc dei voti ${ }^{76}$. Orbene, in tutti i paesi nei quali l'elettore può scegliere, i candidati possono competere, e i risultati non possono essere falsati, in tutti questi paesi le elezioni libere sono «monofunzionali », e cioè assolvono una stessa funzione primaria: quella di consentire all'elettorato di insediare o sostituire i propri governanti. Quando e dove le elezioni servono ad altri scopi, non sono strutturate allo stesso modo. Ergo non è vero che le elezioni siano multifunzionali: è vero, all'opposto, che per funzionare diversamente richiedono una diversa struttura.

Il verdetto non è, allora, che lo struttural-funzionalismo, giungendo a conclusioni suicide, ha dimostrato la propria superfluità. Il verdetto è, piuttosto, che l'approccio sta girando a vuoto: sta girando a vuoto sia perché la diade non c'è, sia perché non acchiappa quel che si era specialmente proposto di acchiappare: le strutture. Finora - riconosciamolo - abbiamo fatto un buco nell'acqua. Ma l'impresa non è da abbandonare: è da perseguire su due gambe, e con gambe migliori. E con questo vengo, o torno, al problema della formazione-malformazione dei concetti.

Finora ho messo in luce le magagne che la scuola strutturale-funzionale ha ereditato, tutto sommato, dal piú generale contesto del funzionalismo ${ }^{n}$. Vediamo ora le difficoltà che la

76 E questo che intendo per «descrizione strutturale» (supra, nota 73). Tale è, nella fattispecie, il vol. di William J. M. Mackenzie, Free Elections, London, Allen \& Unwin, 1958, che l'A. dichiara un « testo elementare »: eppure gli occorrono 180 pagine fitte.

77 Senza risalire per questo agli antropologi, basti ricordare la trop- 
scuola si è creata con le proprie mani, e cioè per cattivo trattamento dei suoi concetti portanti.

Ricominciamo da «funzione ». Per quanto si possa ritenere che questo concetto sia, di per sé, un costrutto teorico, la determinazione e classificazione delle funzioni - le funzioni in concreto - sono una partita che riguarda la scala di astrazione e una corretta tecnica classificatoria ${ }^{78}$. E qui davvero non ci siamo. Per rendersene conto a prima vista basta prendere l'ultimo elenco delle funzioni sistematiche di Almond, raggruppate in a) funzioni di conversione (articolazione degli interessi, aggregazione degli interessi, comunicazione politica, e formazione, applicazione e somministrazione delle norme), $b$ ) capacità (estrattiva, regolativa, distributiva, simbolica e sensitiva), c) funzioni di mantenimento e adattamento (reclutamento e socializzazione politica); e poi vedere come questa disparata congerie di funzioni viene distribuita e calata, in concreto, a livello subsistemico, e cioè nei tre maggiori sotto-sistemi da lui considerati: gruppi di interesse, partiti, governo ${ }^{79}$. Il guazzabuglio è inimmaginabile, e certo non può essere districato qui. Mi limito ad estrarre dal mucchio il caso della funzione che ho appositamente indicato in corsivo: la funzione comunicativa, o funzione di « comunicazione politica ». Salta all'occhio che questa è la categoria piú astratta e onnicomprensiva di tutta la serie. Almond stesso riconosce, ad un certo punto, che è il « prerequisito necessario » di tutte le altre funzioni ${ }^{80}$. Pertanto i casi sono due: o si fa di «comunicazione » la categoria universale per eccellenza che decifra tutto il resto, come in Deutsch e, in generale, nell'approccio cibernetico ${ }^{81}$; oppure la si sottende

po supina ricezione di Merton, di Talcott Parsons, e di Marion J. Levy jr. (il piú influente divulgatore della scoperta della multi-funzionalità). $\mathrm{Su}$ questi antefatti prossimi vedi Walter Buckley, Structural-Functional Analysis in Modern Sociology, nel vol. coll. curato da H. Becher e A. Boskoff, Modern Sociological Theory in Continuity and Change, New York Dryden Press, 1957.

(78) La scala di astrazione costituisce, pertanto, lo strumento per stabilire le «regole di corrispondenza » (in senso carnapiano) di «funzione » (supra, nota 62): e io direi addirittura le regole di trasformazione che la rendono riconducibile ad osservazioni.

79 Almond e Powell, Politica comparata, cit., pp. 47-52 e passim. Il corsivo è mio.

80 Ibidem, p. 225.

81 Di Deutsch vedi spec. The Nerves of Government, New York, Free Press, 1963. In generale, cfr. Richard R. Fagen, Politics and Communication, Boston, Little Brown, 1966. 
e richiama di volta in volta a titolo di variabile interveniente. Quel che non si può fare è quel che Almond ne fa: giustapporla, in un qualche angolino, accanto a funzioni alienum genere che palesemente appartengono, tra l'altro, ad altro livello di astrazione ${ }^{82}$.

Prescindendo da Almond, prendiamo un esempio piú maneggevole, e per ciò stesso a livello sub-sistemico: le funzioni dei partiti (del sotto-sistema partitico). In altra sede ne ho contate - senza alcuna pretesa di aver esaurito la letteratura ventisette: tante per un solo "segmento ${ }^{83}$. Eppure, a pensarci bene, una trentina di funzioni non sono molte se teniamo presente la grande varietà dei sistemi partitici, e cioè la molteplicità di «strutture partitiche » alle quali queste funzioni fanno capo ${ }^{84}$. Qual è il problema? Ovviamente una mera enumerazione casuale di una trentina di funzioni non serve a niente, e semmai aggiunge alla confusione. Occorre sistematizzarle e vagliarle alla luce di almeno tre criteri: 1 ) di organizzazione verticale, 2) di corrispondenza tra funzioni e strutture, 3) di fungibilità.

1) Il primo criterio - verticale - ci impone di collocare le categorie funzionali ai diversi livelli di astrazione ai quali competono. A questo modo scopriamo, per esempio, che la funzione di comunicazione (dei partiti) pecca di genericità (all'alto livello), e quindi che occorre specificarla scendendo per tutta la scala di astrazione: quale tipo, e poi quali sottospecie di comunicazione? A questo modo scopriamo anche, in secondo luogo, che talune funzioni si incastrano verticalmente, talché non vanno giustapposte l'una accanto all'altra (come se fossero 《altre ») ma aggregate o disaggregate a seconda della specificità dell'analisi. Si tratta, in definitiva, di una stessa funzione che varia - inversamente - per denotazione e connotazione. Per

${ }^{82}$ Basti notare che l'articolazione e l'aggregazione degli interessi si definiscono per esclusione reciproca, mentre comunicazione rischia di non avere un contrario. Cosa è, infatti, non-comunicazione?

83 Cfr. Concept Misformation in Comparative Politics, loc. cit., nota 54.

84 Nella mia Tipologia dei sistemi di partito, in «Quaderni di Sociologia », XVII (1968), pp. 187-226, propongo una tassonomia di base a sette voci [sistemi: 1) a partito unico, 2) a partito egemonico, 3) a partito predominante, 4) bipartitici, 5) di multipartitismo moderato, 6) di multipartitismo estremo, 7) atomizzati] che peraltro arriva subito, con le sottoclassi, a 10 voci (v. spec. pp. 225-226). Aggiungi che questa tipologia non include le situazioni fluide di gran parte del terzo mondo. 
esempio, le funzioni di «far partecipare » e/o di «mobilitare » un elettorato sono sottoclassi della classe electioneering, e cioè della funzione di attivazione elettorale: cosicché quest'ultima classe sta sopra, non accanto alle altre due. Infine, e in terzo luogo, una tassonomia che si dispiega lungo una scala di astrazione ci aiuta a disaccavallare funzioni troppo contigue, che si sovrappongono senza essere per questo coestensive. E il caso della funzione di integrazione, che si accavalla con quelle dette di coesione e di mantenimento del consenso. Ė, ancora, il caso delle funzioni variamente dette di mediazione, moderazione, riconciliazione: se sono tre, rendiamole tali; se non ci riusciamo, rendiamole omologhe e contiamole per una.

2) Il secondo criterio - cercare le corrispondenze - presuppone che tanto le funzioni quanto le strutture siano debitamente articolate e classificate, ognuna per suo conto. Dopodiché le due serie debbono essere messe in corrispondenza per stabilire come combaciano: quali funzioni corrispondono a quali strutture partitiche, e viceversa. E la mia congettura è che a questo punto scopriremo semmai che le nostre trenta funzioni non bastano.

3) Il terzo criterio - di fungibilità - consegue al secondo e consente di sistemare la questione pendente della multi-funzionalità. Quali sono le funzioni davvero multi-strutturali, e cioè espletate da qualsiasi struttura? E quali sono, all'altro estremo, le funzioni uni-strutturali, e cioè non fungibili, legate ad una sola struttura? Infine, quali sono - nella gamma dei casi intermedi - le funzioni compatibili con, mettiamo, cinque strutture, ma incompatibili con altre cinque? A questo modo le funzioni diventano scalabili secondo un ordine di minore o maggiore fungibilità. A lume di naso direi, per fare tre esempi, che la funzione piú fungibile - e cioè espletata in modo tollerabilmente assimilabile da tutte le strutture partitiche - risulterebbe quella di canalizzazione. La funzione espressiva (della domanda) si collocherebbe verso la metà della scala, se è vero che compare in tutte le strutture pluralistiche e competitive, ma scompare in quelle monistiche. E una funzione come quella della semplificazione delle alternative rischierebbe di risultare tra le meno fungibili, e cioè di restare confinata alle strutture bipartitiche.

Dicevo che - ad effetto della determinazione e classificazione delle funzioni - davvero non ci siamo. Difatti nella 
letteratura non c'è quasi traccia di un trattamento del tipo delineato sopra: né per le funzioni dei partiti ${ }^{85}$, né per le funzioni di altri sottosistemi. Nella letteratura regna un caos di enumerazioni a casaccio, di denominazioni funzionali che si accavallano, e di acrobatici salti tra funzioni onnicomprensive, astratte al punto da essere vaporizzate, e funzioni di dettaglio. E non andiamo meglio - passando all'altro termine della diade in sede di strutture.

Sul versante delle strutture sappiamo che le attenuanti oggettive sono maggiori. Il punto resta, comunque, che anche il concetto di struttura è stato « mal trattato », e cioè mal formato. In primissimo luogo, struttura a quale livello? Il quesito verticale non è sollevato; dal che consegue che il concetto resta altamente indefinito, e davvero malamente definito ${ }^{86}$. $\mathrm{E}$ vediamo subito che cosa ci potrebbe dire al riguardo una proiezione lungo la scala di astrazione.

Svolgendo «struttura » dall'alto in basso, si possono identificare a prima vista almeno quattro livelli di utilizzazione del termine: struttura intesa come 1) principii strutturali (ad es. pluralismo); 2) condizioni strutturali (ad es. la struttura economica di classe e simili); 3) moduli strutturali quali la strutturazione dei processi elettorali, di opposizione, di pressione, e simili; 4) specifiche strutture organizzative dei membership systems (piú o meno corrispondenti all'organigramma e agli statuti).

Nel primo senso, il piú rarefatto, le strutture sono soltanto $\mathrm{i}$ 《 principii » che presiedono alla convivenza e alla articolazione degli aggregati umani entro una qualche forma politica. Anche

85 Cfr. a conferma, Howard A. Scarrow, The Functions of Political Parties: A Critique of the Literature and the Approach, in «Journal of Politics », XXIX (1967), pp. 770-790. In questa rassegna critica, sottile per altri rispetti, i problemi di cui sopra non sono nemmeno intravisti.

s6 Cfr. per tutti Almond: « per struttura intendiamo le attività osservabili che compongono il sistema politico. L'accennare a queste attività come aventi una struttura implica semplicemente che esse si verificano con una certa regolarità » (Politica comparata, cit., p. 59). Si noti, per inciso, che anche le funzioni sono, per Almond, «attività » (mai meglio definite). Subito dopo Almond cambia definizione, forse nel tentativo di stringere qualcosa di piú: «per strutture intendiamo quei particolari insiemi di ruoli che sono collegati tra loro » (ibidem). La preoccupazione behavioristica è evidente. Altrettanto evidente è il risultato paradossale al quale approda il behaviorismo: di rendere inosservabile persino l'osservabile. Con un concetto di struttura configurato come in Almond, tutto e nulla sono strutture. 
il secondo senso è, per l'analisi strutturale-funzionale, una condizione di sfondo - un parametro - piú che una struttura di primario interesse. In concreto, le strutture da afferrare sono quelle del terzo e quarto gradino di astrazione. $\mathrm{E}$ se non si afferrano quelle strutture, ci sfugge il piú. Come ci è sinora sfuggito. Beninteso, la rilevazione dei moduli strutturali che danno forma ai processi politici, quali i processi di opposizione e di pressione, è spesso impresa difficilissima, e certo la materia è sfuggente ${ }^{87}$. Non è nemmeno detto che la rilevazione della struttura reale dei membership systems sia impresa di tutto riposo, visto che la struttura legale delle organizzazioni non ci dice mai tutto, e ci può anche mettere del tutto fuori strada. $\mathrm{Ma}$ se nessuno nega le difficoltà di arrivare ad una adeguata e sufficiente «descrizione strutturale », il fatto resta che questa determinazione ci sfugge per una ragione pregiudiziale: per difetto di concetto, e cioè perché non ci viene nemmeno chiesto di cercarla. Al principio di tutto sta la formazione - o mal formazione - del concetto.

La mia conclusione si salda, con ciò, alla mia premessa maggiore: che il punto di maggior debolezza della politica comparata - sub specie di analisi funzionale-strutturale - è da ricondurre ad una inconsapevolezza dei procedimenti astraenti, tale che la scala di astrazione viene non solo ignorata, ma inavvertitamente distrutta nel corso di una troppo precipitosa arrampicata verso categorie onnicapienti ${ }^{88}$. Ma questo non è un vizio costitutivo dell'approccio. Ne è, piuttosto, un vizio di fretta e di crescenza.

${ }^{87} \mathrm{Ma}$ vedrei benissimo la analisi di Dahl sulle condizioni, siti e strategie delle opposizioni, ritrascritta in chiave di «strutturazione delle opposizioni ». Cfr. nel vol. da lui curato, Political Oppositions in Western Democracies, New Haven, Yale University Press, 1966, i capp. 11-12-13. Analogamente, le numerose ricerche sui gruppi di pressione potrebbero essere rivisitate, non senza frutto, in chiave strutturale.

${ }_{88}$ Sulla piú generale debolezza metodologica e logica dell'approccio, e per altre severe critiche, cfr. Robert E. Dowse, A Functionalist's Logic, in «World Politics », XVIII (1966), pp. 607-622; e Arthur L. Kalleberg, The Logic of Comparison, in «World Politics», XIX (1966), pp. 69-82. 
Riepilogo.

La scienza politica che oggi dichiariamo pre-scientifica aveva - con tutti i suoi errori e difetti - una fecondità teoretica; fecondità teoretica che la scienza politica «scientificizzata » di ispirazione behavioristica ha in gran parte perduta, o quantomeno atrofizzata ${ }^{89}$. Per dire meglio - con maggiore rigore ed esattezza - diciamo meno. Ai grandi problemi, dichiarati scientificamente intrattabili, sottentrano i micro-problemi, che a loro volta generano il puro virtuosismo tecnico: la micro-analisi senza problema. È un prezzo molto alto. Occorre davvero pagarlo? Forse no. E certo non occorre pagarlo nella misura in cui la macro-analisi, e per essa anche l'analisi dei grandi problemi, è riconducibile a ipotesi, generalizzazioni e previsioni verificabili, o comunque falsificabili.

Da qui l'importanza crescente della politica comparata, che oggi si colloca al centro della scienza politica contemporanea. Comparare è controllare. Pertanto è nella politica comparata che la scienza politica ritrova - ad un piú alto livello di consapevolezza scientifica e di validità empirica - i grandi problemi, e ritrova con essi e per essi la sua fecondità teoretica. Ma l'impresa di una politica comparata che investe la globalità, e che se ne serve a fini di controllo, è davvero una impresa di lunga lena: tanto inedita e innovatrice, quanto tentativa e rischiosa. Non può stupire, dunque, che $\mathrm{i}$ frutti siano ancora acerbi e

${ }^{89}$ Non sempre, e non necessariamente, beninteso. Per l'altra faccia della medaglia cfr. il vol. cit. curato da Easton, Varieties of Political Theory, e la sezione Political Theory nel vol. cit. Approaches to the Study of Political Science (pp. 51-121). Ma vedi soprattutto il caso di Robert A. Dahl, un autore che nel pieno rispetto dei canoni del behaviorismo, e anzi in chiave operazionale, è riuscito a darci, con A Preface to Democratic Theory, Chicago, Univ. of Chicago Press, 1956, il libro teoreticamente piú puntuale e penetrante di tutta la letteratura sulla democrazia. Ai nostri fini non è senza interesse notare che anche Dahl si è andato man mano spostando sul terreno comparato: e questo non solo nel volume cit. Political Oppositions in Western Democracies (al quale segue un secondo volume sulle opposizioni fuori dal contesto democratico), ma anche nel progetto su The Politics of the Smaller European Democracies, di prossima pubblicazione, di cui Dahl è il coordinatore. Il che aggiunge, ma non sposta, ad effetto della fecondità e finezza teoretica di un autore in apparenza cosí alieno della teorizzazione. Questo filo nascosto, e per esso la statura intellettuale di Dahl, è colto da Domenico Fisichella, Temi e metodi in scienza politica, Firenze, Sansoni, 1971, cap. VI. 
spesso indigesti. Non è ancora vero, nel nostro caso, che «la comparazione è la base della creatività e della crescita cumulativa della scienza ${ }^{90}$. Ma non ci dobbiamo scoraggiare per questo. E se il mio riesame critico della « nuova » politica comparata alla luce delle sue premesse logiche e metodologiche risulta un esame severo, la mia critica vuol essere costruttiva, non certo negativa e negatrice.

Le ambizioni globali della nuova politica comparata sollevano spinosi e inediti problemi metodologici di fondo, problemi che restano da affrontare e che non sono stati nemmeno adeguatamente percepiti. E se la messa a fuoco di questo saggio è concettuale - sui concetti - si deve intendere che $i$ concetti in questione non sono soltanto elementi di un sistema teoretico ma sono anche, e del pari, strumenti di ricerca e contenitori di dati.

Il problema empirico si imposta cosí: ci mancano informazioni abbastanza precise da essere significativamente e sicuramente paragonabili. Di conseguenza abbiamo estremo, urgente bisogno di un sistema standardizzato di rilevazione-schedatura costituito da contenitori concettuali discriminanti, resi tali da una tecnica di scomposizione tassonomica. L'alternativa è il datamisgathering, il mettere assieme dati mal reperiti: con il che rischiamo di essere travolti $\mathrm{da}$ una disinformazione rinforzata dal trattamento automatico, alla quale nessuna rigenerazione statistica, per quanto raffinata, può rimediare.

Il problema teorico, o teoretico, si imposta, correlativamente, cosí: ci occorrono regole atte a disciplinare il vocabolario e i procedimenti di comparazione. Altrimenti rischiamo di naufragare nel caos e nella frivolità di vuote assimilazioni e generalizzazioni. In particolare l'indisciplina nell'uso dei termini e dei procedimenti di comparazione ha per corrispettivo il cattivo trattamento dei concetti e approda, in ultima istanza, ad una vera e propria disformazione del concetto (disformazione che si salda senza soluzione di continuità con la disinformazione).

Il filo conduttore di una qualche, necessarissima disciplina è stato da me indicato nella scala astraente, nelle proprietà logiche dei relativi livelli di astrazione, e nelle regole di percorrimento, di composizione e scomposizione: regole di percorrimento che ci consentono di congiungere un forte potere espli-

${ }^{90}$ Michael Haas, Comparative Analysis, in «Western Political Quarterly », XV (1962), p. 303. 
cativo e generalizzante, con un contenuto descrittivo suscettivo di verifica empirica. Beninteso, non si tratta di una ricetta magica, e nemmeno di una ricetta applicabile a tutti i problemi ${ }^{91}$. $\mathrm{Ma}$ certo lo schema di riferimento della scala di astrazione introduce ordine nel caos, ci salva dallo «stiramento del concetto », e anzi ci porta a sviluppare con metodo un vocabolario piú analitico. Si potrà osservare che, a rigore, i livelli di analisi non sembrano convertibili l'uno nell'altro senza residui; e dunque che, salendo o scendendo lungo la scala di astrazione, c'è sempre qualcosa che si perde o si acquista. Difatti la disposizione verticale dei concetti non è continua: e si dice scala anche e proprio per richiamare l'immagine dei gradini. $\mathrm{Ma}$ anche ammettendo una irriducibilità 《perturbante », resta vero che la disciplina imposta dalla scala di astrazione e dalle sue regole fa sí che le asserzioni teoretiche generate da un particolare livello trovino, nei livelli circostanti, asserzioni atte a confermarle o a contraddirle.

Un'ultima considerazione. Può sembrare che il mio discorso combini assieme due difetti: quello di essere troppo approssimativo e superficiale per chi s'intende di filosofia analitica e di metodologia della scienza; e quello di essere, viceversa, troppo esigente e filosofico per il contesto in cui si situa, e cioè per il politologo. $\mathrm{Ma}$ se questi sono difetti, sono da me scientemente coltivati. Perché il mio intendimento è proprio di avvicinare due posizioni estreme che ritengo egualmente perniciose, e tra mezzo alle quali sta un immenso vuoto protetto da un muro di incomunicabilità. Scriveva Wright Mills: « dominare la "teoria" e il "metodo" equivale a diventare un pensatore consapevole, un uomo che lavora sapendo quali sono i presupposti e le implicazioni di quel che fa. Essere dominati dalla "teoria" e dal "metodo" equivale a non cominciare mai a lavorare $»^{92}$. La mia linea è quella di Mills. Io credo, con lui, al « pensatore consapevole », egualmente alieno dal perfezionismo iper-con-

91 Resta aperto, per es., il problema di come l'analisi a livello degli Stati si rapporta all'analisi a livello internazionale. Cfr. J. David Singer, The Level-of-Analysis Problem in International Relations, nel vol. curato da K. Knorr e S. Verba, The International System, Princeton, Princeton University Press, 1961, spec. pp. 91-92. Ma questo è un problema di come saldare due o piú discipline che adoperano categorie diverse.

92 Wright Mills, On Intellectual Craftmanship, in Gross (ed.), Symposium on Sociological Theory, cit., p. 27. Il corsivo è mio. 
sapevole e dal semplicismo inconsapevole. Al perfezionista (alla Hempel) che rifiuta di considerare il caldo e il freddo se, e finché, non dispone di un termometro, rimprovero di applicare troppo spesso alle scienze sociali un metro non pertinente e controproducente. $\mathrm{Ci}$ piaccia o non ci piaccia, le scienze dell'uomo nuotano tuttora in un mare di ingenuità: e il piú ingenuo di tutti finisce per essere chi crede di essere approdato in terra ferma. Con il che - dovrebbe essere chiaro da tempo non intendo in alcun modo redimere il «pensatore inconsapevole », chi pensa senza addestramento al pensare. 